\title{
Atrophy and Death of Nonpeptidergic and Peptidergic Nociceptive Neurons in SIV Infection
}

Jake A. Robinson, * Guy Guenthner, ${ }^{\dagger}$ Rebecca Warfield, ${ }^{*}$ Jessica R. Kublin, ${ }^{\dagger}$ Mandy D. Smith, * Masoud Shekarabi, * Andrew D. Miller, ${ }^{\ddagger}$ and Tricia H. Burdo*

From the Department of Neuroscience, ${ }^{*}$ Temple University, Lewis Katz School of Medicine, Philadelphia, Pennsylvania; the Department of Biology, ${ }^{\dagger}$ Boston College, Chestnut Hill, Massachusetts; and the Section of Anatomic Pathology, ${ }^{\ddagger}$ Department of Biomedical Sciences, Cornell University College of Veterinary Medicine, Ithaca, New York

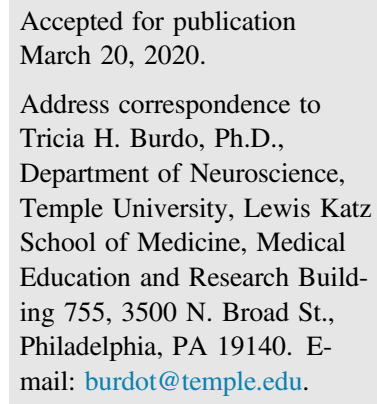

\begin{abstract}
HIV-associated sensory neuropathy is a common neurologic comorbidity of HIV infection and prevails in the post-antiretroviral therapy (ART) era. HIV infection drives pathologic changes in the dorsal root ganglia (DRG) through inflammation, altered metabolism, and neuronal dysfunction. Herein, we characterized specific neuronal populations in an SIV-infected macaque model with or without ART. DRG neuronal populations were identified by neurofilament $\mathrm{H}$-chain $200, \mathrm{I}_{4} \mathrm{~B}_{4}$ isolectin (IB4), or tropomyosin receptor kinase $A$ expression and assessed for cell body diameter, population size, apoptotic markers, and regeneration signaling. $\mathrm{IB}^{+}$and tropomyosin receptor kinase $\mathrm{A}-$ positive neurons showed a reduced cell body size (atrophy) and decreased population size (cell death) in the DRG of SIV-infected animals compared with uninfected animals. IB4 ${ }^{+}$nonpeptidergic neurons were less affected in the presence of ART. DRG neurons showed accumulation of cleaved caspase 3 (apoptosis) and nuclear-localized activating transcription factor 3 (regeneration) in SIV infection, which was significantly lower in uninfected animals and SIV-infected animals receiving ART. Nonpeptidergic neurons predominantly colocalized with cleaved caspase 3 staining. Nonpeptidergic and peptidergic neurons colocalized with nuclear-accumulated activating transcription factor 3 , showing active regeneration in sensory neurons. These data suggest that nonpeptidergic and peptidergic neurons are susceptible to pathologic changes from SIV infection, and intervention with ART did not fully ameliorate damage to the DRG, specifically to peptidergic neurons. (Am J Pathol 2020, 190: 1530-1544; https://doi.org/10.1016/j.ajpath.2020.03.004)
\end{abstract}

With the introduction of combined antiretroviral therapy (ART), HIV infection has transitioned to a chronic disease, shifting focus to HIV-associated comorbidities, persistent inflammation, and early-onset age-related illnesses. HIVassociated sensory neuropathy remains one of the most common neurologic complications of HIV infection, significantly contributing to HIV morbidity with little reduction in prevalence from ART. ${ }^{1-4}$ The most common form of HIV-associated sensory neuropathy, HIV-associated distal sensory polyneuropathy (HIV-DSP), develops from damage to peripheral sensory neurons of the dorsal root ganglia (DRG) and the dying back of the primary afferent axon terminals. ${ }^{5-7}$ HIV-DSP manifests with pain, numbness, tingling sensations, and temperature hypersensitivity in the extremities as a result of abnormal peripheral neuron function; however, the pathophysiology of neuronal damage and dysfunction is multifaceted and not fully understood.

The DRG are located adjacent to the spinal column and consist of a heterogeneous population of somatosensory neurons responsible for conveying peripheral sensations to the central nervous system through innervating the dorsal horn of the spinal cord. Of particular interest in HIV-DSP,

\footnotetext{
Supported by NIH grants R01 NS082116 (T.H.B.), P30 MH092177 (T.H.B.), and T32 MH079785 (J.A.R., R.W.); and Tulane National Primate Research Center's base grant P51OD011104 for SIV ${ }^{-}$tissues and SIVmac251 viral stocks. The in vivo CD8-depletion antibodies used in these studies were purchased from the NIH Nonhuman Primate Reagent Resource under grants RR016001 and AI040101.

Disclosures: T.H.B. holds equity in Excision BioTherapeutics unrelated to this article. No writing assistance was used in the production of this article.
} 
C-fiber nociceptive neurons make up most of the somatosensory neurons in the DRG and are responsible for conveying pain, temperature, itch, and touch. ${ }^{8-10}$ Nociceptive neurons have been classified as nonpeptidergic, identified as $\mathrm{I}_{-} \mathrm{B}_{4}$ isolectin (IB4) binding neurons, and peptidergic neurons, expressing tropomyosin receptor kinase A (TrkA) and neuropeptides, such as calcitonin gene-related peptide. ${ }^{10-13}$ Nociceptive afferents in cutaneous tissue are mostly unmyelinated and branch into the epidermal area as free-end terminals. ${ }^{14-17}$ Innervating free axon terminals are particularly susceptible to changes in inflammatory status, as they can directly interact with macrophages, neutrophils, mast cells, Merkel cells, and blood vessels in the skin. In the presence of pathologic changes and disease, nerve fibers die back from the epidermal layer as a result of damage to terminals, axons, and cell bodies.

DRG neurons are long-lived neurons with high axonal regenerative capacity and resilience to environmental changes; however, death of DRG neurons is permanent. In the presence of HIV infection, neurons have diminished regenerative capacity, impaired pain transmission, and abnormal metabolic function, all contributing to loss of axon terminals innervating the periphery. ${ }^{18-21}$ Damage to peripheral neurons is accompanied with significant transcriptional changes in survival genes and regeneration-associated genes. ${ }^{22,23}$ An immediate-early gene in the regenerative program is activating transcription factor 3 (ATF3). The increased expression and nuclear accumulation of ATF3 is integral to the initiation and maintenance of the regenerative state of peripheral neurons. ${ }^{24-27}$ ATF3 was found to be a critical mediator of regeneration-associated gene expression in sensory neurons and promoted significant neurite regrowth. ${ }^{28,29}$ Priming neurons for regeneration is dependent on transcriptional changes from multiple, converging pathways and vital for sensory neuron survival in challenge.

The extent of damage to sensory neurons remains unclear in the context of HIV infection. Previous studies in animal models have detailed the presence of viral proteins in the DRG, satellite cell activation, increased infiltration of mononuclear cells into the DRG, and increased production of inflammatory mediators in the DRG, detailing a prominent role of inflammation in development of pathology. ${ }^{18,21,30-32}$ However, specific neuronal population changes and damage responses have yet to be investigated. The SIV-infected rhesus macaque model was used to characterize alterations in different sensory neuron populations, the induction of regeneration transcription factors, and activation of apoptosis in the DRG, to infer neuronal contributions to and exacerbation of the clinical manifestations of HIV-DSP. A significant, class-specific atrophy and loss of nociceptive sensory neurons was observed with SIV infection. Both proregenerative, stress-induced signaling (ATF3) and apoptotic signaling (cleaved caspase 3) was observed in DRG neurons during SIV infection. Herein, we show dramatic changes in DRG neurons that suggest, in addition to inflammatory and viral alterations in the DRG, the presence of SIV infection promotes a severe pathologic environment for nociceptive sensory neurons.

\section{Materials and Methods}

\section{Animals, Viral Infection, CD8 T-Cell Depletion, and ART Regimen}

Indian, male rhesus macaques (Macaca mulatta) were used in this study as a model of HIV infection (Table 1). ${ }^{33-36}$ Primates are recognized as an acutely scarce resource and as such are specifically exempted from the requirement of sex balancing; herein, we conducted the study in an all-male cohort (NIH: NOT-OD-15-102). Animals were housed at either the Tulane National Primate Research Center (Covington, LA) or the New England Regional Primate Center. Animals A01 to A08 served as uninfected controls for the study (referred to as SIV $; n=9$ ). Two $\mathrm{SIV}^{-}$animals were CD8 depleted (A01 and A02) and six were not CD8 depleted (A03 to A08). Twenty animals were inoculated intravenously (i.v.) with SIVmac251 viral swarm (5 ng p27; Tulane National Primate Research Center's Viral Core) and subsequently CD8 depleted through administration of 10 $\mathrm{mg} / \mathrm{kg}$ of anti-CD8 antibody subcutaneously at 6 days postinfection (dpi) and $5 \mathrm{mg} / \mathrm{kg}$ of antibody intravenously at 8 and 12 dpi (Nonhuman Primate Reagent Resource), as previously described. ${ }^{21,30,37}$ Animals A09 to A11 were time sacrificed at $21 \mathrm{dpi}$ on establishment of viral infection (referred to as SIV/DPI21; $n=3$ ). Animals A12 to A22 did not receive a treatment regimen and progressed to terminal SIV and simian AIDS (referred to as SIV/AIDS; $n=11$ ). The development of simian AIDS was determined postmortem, as previously described. ${ }^{21,30,37}$ Animals A23 to A28 received a clinically relevant ART regimen of raltegravir $(22 \mathrm{mg} / \mathrm{kg}$ orally twice daily; Merck, Kenilworth, $\mathrm{NJ}$ ), tenofovir (30 mg/kg subcutaneously once daily; Gilead, Foster City, CA), and emtricitabine $(10 \mathrm{mg} / \mathrm{kg}$ subcutaneously once daily; Gilead) at $21 \mathrm{dpi}$ until the timed sacrifice at 118 to $120 \mathrm{dpi}$ (referred to as SIV + ART; $n=6$ ). ${ }^{38,39} \mathrm{~A}$ representative experimental design details SIV inoculation, CD8 depletion, and ART administration for all animals (Supplemental Figure S1). All animals were anesthetized with ketamine-HCL and euthanized by i.v. pentobarbital overdose. These animals were sacrificed according to humane end points consistent with the recommendations of the American Veterinary Medical Association Guidelines for the Euthanasia of Animals, as previously described. ${ }^{21,37,40}$ Animals were necropsied immediately following death, and representative sections of all major organs were collected for histologic analysis. All animals used in this study were handled in strict accordance with American Association for Accreditation of Laboratory Animal Care with the approval of the Institutional Animal Care and Use Committee of Harvard University and the Institutional Animal Care and Use Committee of Tulane University. 
Table 1 Viral, Pathologic, and Zoologic Information of Animals Used in the Study

\begin{tabular}{|c|c|c|c|c|c|c|}
\hline Group I.D. & Animal I.D. & Age, years & Primate center & Survival, days & $\begin{array}{l}\text { Plasma VL, } \log _{10} \\
\text { copies } / \mathrm{mL}^{*}\end{array}$ & DRG pathology (score $\left.{ }^{\dagger}\right)$ \\
\hline \multirow[t]{5}{*}{ Uninfected (SIV-) } & A01 & 6.0 & NERPC & N/A & $\mathrm{N} / \mathrm{A}$ & Normal (0) \\
\hline & A03 & 10.8 & TNPRC & N/A & $\mathrm{N} / \mathrm{A}$ & $\mathrm{N} / \mathrm{A}$ \\
\hline & A04 & 9.6 & TNPRC & N/A & $\mathrm{N} / \mathrm{A}$ & $\mathrm{N} / \mathrm{A}$ \\
\hline & A05 & 6.7 & TNPRC & $\mathrm{N} / \mathrm{A}$ & $\mathrm{N} / \mathrm{A}$ & $\mathrm{N} / \mathrm{A}$ \\
\hline & A08 & 3.3 & TNPRC & N/A & N/A & $\mathrm{N} / \mathrm{A}$ \\
\hline \multirow{3}{*}{$\begin{array}{l}\text { SIV infected and CD8 } \\
\text { depleted (SIV/DPI21) }\end{array}$} & A09 & 9.1 & TNPRC & 21 & 6.87 & Moderate (2) \\
\hline & A10 & 5.2 & TNPRC & 21 & 7.15 & Moderate (2) \\
\hline & A11 & 5.3 & TNPRC & 21 & 6.84 & Mild (1) \\
\hline & A15 & 11.5 & TNPRC & 146 & 7.67 & Severe (3) \\
\hline & A16 & 3.8 & NERPC & 77 & 7.23 & Moderate-severe (2.5) \\
\hline & A17 & 5.8 & NERPC & 77 & 8.54 & Moderate (2) \\
\hline & A18 & 4.3 & NERPC & 168 & 5.88 & Mild (1) \\
\hline & A19 & 5.4 & NERPC & 97 & 7.79 & Mild (1) \\
\hline & A20 & 6.3 & TNPRC & 84 & 5.41 & Severe (3) \\
\hline & A21 & 4.5 & TNPRC & 96 & 7.15 & Mild (1) \\
\hline & A22 & 7.3 & TNPRC & 106 & 7.69 & Moderate (2) \\
\hline \multirow{2}{*}{$\begin{array}{l}\text { SIV infected and ART } \\
\text { treated (SIV + ART) }\end{array}$} & A23 & 6.1 & TNPRC & 118 & 2.66 & Mild (1) \\
\hline & A24 & 6.7 & TNPRC & 118 & 2.66 & Mild (1) \\
\hline
\end{tabular}

All animals in the study were male, Indian rhesus macaques.

* Plasma VL was measured at necropsy as terminal viral load.

${ }^{\dagger}$ General scoring includes lumbar and sacral DRG for pathology score. Pathologic grading and criteria based on evaluation from a certified veterinary pathologist (refer to Materials and Methods).

DRG, dorsal root ganglia; I.D., identifier; N/A, not applicable; NERPC, New England Regional Primate Center; Plasma VL, plasma viral load, at necropsy; TNPRC, Tulane National Primate Research Center.

\section{Pathologic Assessment}

Tissues were evaluated for pathologic changes in the DRG through hematoxylin and eosin staining. Sections were evaluated blindly by a board-certified veterinary anatomic pathologist (A.D.M.) and scored on the basis of the presence and severity of infiltrating mononuclear cells, neuronophagia, and Nageotte nodules, as previously described. ${ }^{21,30,31,33}$ Overall pathology was scored on a previously validated scale of 1 to 3 at increments of 0.5 via the following criteria: i) mild, scattered infiltrating mononuclear cells with rare evidence of neuronophagia and/or neuronal loss; ii) moderate, increased numbers of infiltrating mononuclear cells with occasional neuronophagia and/or neuronal loss; and iii) severe, abundant infiltrating mononuclear cells and frequent neuronophagia and neuronal loss were all present. $^{21,30,31,33}$

\section{Immunohistochemistry}

Tissues were prepared and divided into sections, as previously reported. ${ }^{21,30,31,33}$ Sections were boiled in antigen unmasking buffers for epitope retrieval (Vector, Burlingame, CA; Dako, Carpinteria, CA; and Perkin Elmer, Waltham, MA) and blocked for appropriate endogenous proteins (horseradish peroxidase, alkaline phosphatase, avidin, and biotin). Tissues were incubated in primary antibodies or biotinylated lectins for 1 hour at room temperature (Table 2). Primary antibodies or lectins were detected through horseradish peroxidase-conjugated secondary antibodies (Dako) or avidin-biotin complex (Vector). Target proteins were visualized through the 3,3'-diaminobenzidine chromogen system (Dako), and sections were counterstained with hematoxylin (Sigma, St. Louis, MO). Tissue sections were imaged using the Keyence BZ-X700 microscope in bright 
Table 2 Antibodies Used in Histologic Analysis

\begin{tabular}{|c|c|c|}
\hline Target & $\begin{array}{l}\text { Antibody isotype } \\
\text { (clone) }\end{array}$ & Company \\
\hline CD68 & Mouse (KP1) & Dako (M0814) \\
\hline CD163 & Mouse (EDHu-1) & $\begin{array}{l}\text { Bio-Rad, Hercules, } \\
\text { CA (MCA1853) }\end{array}$ \\
\hline Mac387 & Mouse (Mac 387) & Dako (M0747) \\
\hline BrdU & Mouse (Bu20a) & Dako (M0744) \\
\hline CD3 & Rabbit & Dako (A0452) \\
\hline NF200 & Mouse (N52) & $\begin{array}{l}\text { Millipore, Burlington, } \\
\text { MA (MAB5266) }\end{array}$ \\
\hline TrkA* & Rabbit & $\begin{array}{l}\text { Abcam, Cambridge, } \\
\text { UK (ab76291) }\end{array}$ \\
\hline ATF3 & Rabbit & Sigma (HPA001562) \\
\hline Cleaved caspase 3 & Rabbit & $\begin{array}{l}\text { Cell Signaling, Danvers, } \\
\text { MA (9661) }\end{array}$ \\
\hline Isolectin B4 & Lectin $^{\dagger}$ & Vector (B-1205) \\
\hline
\end{tabular}

*TrkA required High pH Target Retrieval (Dako) for antigen unmasking. ${ }^{\dagger}$ Not an antibody, Biotinylated Griffonia Simplicifolia Lectin I (GSL I) IB4. ATF3, activating transcription factor 3; BrdU, bromodeoxyuridine.

field for 3,3'-diaminobenzidine chromogenic development (Keyence, Osaka, Japan). Histologic stains were quantified using the Keyence BZX Advanced Analysis Software. Hematoxylin was used to determine total number of neurons in individual images, and identification of positive neurons was achieved by threshold of staining intensity through BZX Hybrid Cell Count (Keyence). Quantifications were reported in percentages of total number of neurons (rather than cells/ $\mathrm{mm}^{2}$ ) to account for differences in number of neurons in each section of lumbar DRG. For each animal, at least eight representative images were quantified for percentage positive cells and averaged to give a representative mean.

\section{RNAscope in Situ Hybridization}

Caspase 3 RNA in DRG neurons was visualized using RNAscope, ${ }^{37}$ according to specifications of the manufacturer (ACDBio, Newark, CA; reference number 43656). Slides were deparaffinized through xylene, washed in 100\% ethanol, and air dried. Sections were treated with heat-induced target retrieval $\left(92^{\circ} \mathrm{C}\right.$ to $\left.100^{\circ} \mathrm{C}\right)$ and incubated with protease $\left(40^{\circ} \mathrm{C}\right)$. The probe was hybridized in a humidity chamber at $40^{\circ} \mathrm{C}$ for 2 hours. Caspase 3 RNA was detected by amplification and chromogenic development using the Alkaline Phosphatase-Red Chromogen kit (ACDBio). Sections were counterstained with hematoxylin, dried at $60^{\circ} \mathrm{C}$, and mounted. Slides were imaged for quantification of RNA signal using Texas-Red filter fluorescence with a Keyence BZ-X700 Microscope. Threshold level of detection for RNAscope was determined through Dap8 negative control probes (bacterial gene) and PPIB positive control probe (low-expression constitutive gene).

\section{Immunofluorescence}

Detection of multiple target proteins was done through immunofluorescence using Opal dyes, according to manufacturer's specifications (Perkin Elmer). Paraffin sections were deparaffinized and rehydrated through graded ethanols. Sections were boiled in AR6 (neutral pH) or AR9 (high $\mathrm{pH}$ ) retrieval buffer (Perkin Elmer), then blocked and incubated with primary antibody at room temperature for 1 hour (Table 2). Primary antibodies or lectins were detected by horseradish peroxidase-conjugated dual mouse-rabbit secondary antibody or avidin-biotin complex, respectively. Antibody complexes were visualized with Opal dyes for $520-$, 570-, or 690-nm wavelength. Slides were then heat treated with AR6 or AR9 retrieval buffer to reduce background, strip sections of primary and secondary antibody, and allow restaining for other target proteins. Following detection of all targets, slides were counterstained with spectral DAPI (Perkin Elmer) and mounted with Prolong Gold antifade mounting media (Vector). Slides were imaged using filter cubed immunofluorescence with the BZ-X700 microscope (Keyence). Positive staining was determined by setting a threshold of detection compared with negative controls using Keyence Hybrid Cell Count. The total count of a neuronal population and overlap were determined using Keyence BZX Advanced Analysis Software. The number of double-positive neurons and total number of the neuronal population of interest were counted and used to determine the percentage double-positive neurons. For a magnification of $\times 20$ (ATF3), at least eight representative images were quantified for percentage positive cells and averaged to give a representative median. For a magnification of $\times 60$ (cleaved caspase 3), at least 20 representative images were quantified for percentage positive cells and averaged to give a representative median.

\section{Neuronal Diameter Measurements}

Tissue sections were stained for neurofilament H-chain 200 (NF200), IB4, and TrkA, imaged at $\times 400$ magnification, and stitched to form a representative image of the areas containing all neuronal bodies of the DRG section. Neuronal diameter was blindly assessed to measure all positive neuronal bodies that contained the neuronal nucleus (J.A.R.), and similarly to immunohistochemistry, the neuronal stain threshold was determined by the Keyence BZX Hybrid Cell Count. The diameter was measured from the longest point-topoint distance of the neuronal body using the Keyence BZX Advanced Analysis Software Measurement Module. The average neuronal diameter was determined for each neuronal population as a composite average of all neurons in the section of lumbar DRG. Neuronal diameters were segregated into $5-\mu \mathrm{m}$ bins, and the frequency of diameters was determined within each $5-\mu \mathrm{m}$ increment.

\section{Enzyme-Linked Immunosorbent Assay}

EDTA plasma was used to determine concentrations of inflammatory biomarkers over the course of SIV infection and treatment. Plasma soluble CD163 (IQ Products, Groningen, 
the Netherlands), soluble CD14 (R\&D Systems, Minneapolis, MN), chemokine (C-C motif) ligand (CCL) 2 (R\&D Systems), and CCL5 (R\&D Systems) were measured by enzyme-linked immunosorbent assay, according to manufacturer's instructions. Measurements were run in duplicate with appropriate positive controls and accepted with a percentage CV of $\leq 25 \%$; samples were repeated if the cutoff was exceeded.

\section{Skin Punch Biopsy and IENFD}

Skin punches ( $3 \mathrm{~mm}$ thick) were taken serially near the sural innervation site just distal to the lateral malleolus. Biopsy specimens were taken for each animal at pre-infection and at various time points after infection to necropsy. Biopsy specimens were processed, as previously described, and evaluated for the degree of innervation. ${ }^{18,20,21,30,41}$ The quantitation delivers a measure of nerve fiber length/volume epidermis [intraepidermal nerve fiber density (IENFD)].

\section{BrdU Administration}

Bromodeoxyuridine (BrdU) was prepared as previously reported. ${ }^{21,37} \mathrm{BrdU}$ was administered by slow bolus i.v. in-

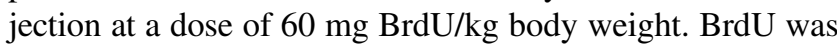
administered at several time points over the course of infections and 24 hours before necropsy in terminal SIV/ AIDS and SIV + ART animals (A12 to A28).

\section{Flow Cytometry}

Flow cytometric analyses were performed with $100-\mu \mathrm{L}$ aliquots of EDTA-coagulated whole blood, as previously reported. ${ }^{21,42,43}$ Erythrocytes were lysed using ImmunoPrep Reagent System (Beckman Coulter, Brea, CA), washed twice with phosphate-buffered saline containing $2 \%$ fetal bovine serum, and then incubated for 15 minutes at room temperature with fluorochrome-conjugated surface antibodies. Samples were acquired on a BD FACS Aria (BD Biosciences, San Jose, CA) and analyzed with Tree Star Flow Jo version 9.6 (BD Biosciences).

\section{Viral Load Measurement}

Plasma SIV-RNA was quantified using real-time PCR for all animals used in this study, as previously described. ${ }^{44}$ The threshold sensitivity was 15 copy $\mathrm{Eq} / \mathrm{mL}$, with an average interassay $\% \mathrm{CV}$ of $<25 \%$.

\section{Statistical Analysis}

All statistics and graphical representations were done using GraphPad Prism version 8.0 (GraphPad, San Diego, CA). Because of small numbers of animals in groups, all comparisons did not assume a gaussian distribution, and a statistical probability value of $P<0.05$ was set for the significance level. All numerical values reported in tables were represented by the median and the interquartile range. All correlations were Spearman correlations and reported with the corresponding $r$ value, if significant. Bar graphs are representative of the median value with the interquartile range. A $U$-test was used in comparisons of two, nonpaired groups. A Kruskal-Wallis test (nonparametric analysis of variance; referred to as analysis of variance in Results and figures) was used to compare three or more, nonpaired groups and, if significant, was followed by a post-hoc Dunn multiple comparison test. Diameter distributions were compared by two-way analysis of variance and, if the interaction was significant, multiple comparisons were tested with a Bonferroni post-test (adjusted for multiple comparisons).

\section{Results}

\section{Animals Used in the Study}

All animals in the study were male, Indian rhesus macaques (Macaca mulatta) (Table 1). Several animals served as uninfected controls in the study (SIV ; A01 to A08; $n=9$ ). SIV-infected animals sacrificed at 21 days postinfection (SIV/DPI21; A09 to A11; $n=3$ ) served as an early time point of established viremia and for comparison for preART initiation. SIV-infected animals that progressed to simian AIDS (SIV/AIDS; A12 to A22; $n=11$ ) provided insight into changes in sensory neurons at a terminal stage of SIV/AIDS. SIV-infected animals that received ART, initiated at 21 days postinfection following the establishment of viremia, were treated with a clinically relevant ART regimen until 118 to 120 days postinfection (SIV + ART; A23 to A28; $n=6$ ). Plasma viral load of SIV + ART animals was successfully decreased several logs by necropsy; however, more important, the viral load was not fully suppressed to undetectable levels and had a median $\log _{10}$ copies $/ \mathrm{mL}$ of plasma of 2.66 [interquartile range $(\mathrm{IQR})=0.90]$ (Table 3 and Supplemental Figure S2). Animals were sacrificed and necropsied for comparison of changes in the DRG as a result of SIV infection.

ART Improves Myeloid Inflammation, DRG Pathology, and DRG Leukocyte Infiltration from SIV Infection

SIV infection generates a severe inflammatory state in the absence of ART. With successful viral load reduction by ART, the necropsy plasma concentrations of SCD14, CCL2, and CCL5 were significantly lower in SIV + ART animals compared with SIV/AIDS animals (plasma markers; $P<0.05, P<0.01$, and $P<0.05$, respectively) (Table 3 ). Plasma sCD163 trended toward a lower plasma concentration in SIV + ART animals compared with SIV/AIDS animals (plasma markers; $P=0.06$ ) (Table 3). The percentage monocytes trended to a lower percentage of peripheral blood mononuclear cells in SIV + ART animals compared 
Table 3 Comparison of Plasma Immune Markers, IENFD, and LDRG Immune Cells of SIV/AIDS and SIV + ART Cohort

\begin{tabular}{|c|c|c|c|c|}
\hline \multirow[b]{2}{*}{ Variable } & \multicolumn{2}{|c|}{ SIV/AIDS } & \multicolumn{2}{|c|}{ SIV + ART } \\
\hline & $n$ & Median (IQR) & $n$ & Median (IQR) \\
\hline \multicolumn{5}{|l|}{ Plasma markers } \\
\hline Soluble CD14, $\mathrm{ng} / \mathrm{mL}$ & 11 & $1358.2(253.8)$ & 6 & $707.2(216.2)^{*}$ \\
\hline Soluble CD163, ng/mL & 11 & $412.4(230.8)$ & 6 & $268.2(103.4)$ \\
\hline $\mathrm{CCL} 2, \mathrm{pg} / \mathrm{mL}$ & 11 & $719.3(912.5)$ & 6 & $118.6(125.7)^{\star *}$ \\
\hline Monocytes, \% & 8 & $5.3(6.9)$ & 6 & $4.0(2.6)$ \\
\hline $\mathrm{CD}_{14}{ }^{+} \mathrm{CD} 16^{+}$monocytes, $\%$ & 8 & $22.3(19.4)$ & 6 & $6.6(5.9)^{* *}$ \\
\hline $\mathrm{CD}_{14}{ }^{-} \mathrm{CD} 16^{+}$monocytes, $\%$ & 8 & $9.3(10.4)$ & 6 & $4.5(3.9)^{*}$ \\
\hline $\mathrm{CD}_{14}{ }^{+} \mathrm{CD} 16^{-}$monocytes, $\%$ & 8 & $63.7(28.2)$ & 6 & $88.8(9.7) * *$ \\
\hline CD163 expression on $\mathrm{CD} 14^{+} \mathrm{CD} 16^{+}$monocytes, MFI & 8 & $22,543.0(18,582.8)$ & 6 & $4751.0(3659.0) * *$ \\
\hline $\mathrm{CD}_{163^{+}}$macrophages & 11 & $1306.0(831.4)$ & 6 & $659.9(395.0) *$ \\
\hline Mac387 ${ }^{+}$monocytes & 11 & $156.7(93.0)$ & 6 & $26.3(40.4)^{* * *}$ \\
\hline $\mathrm{BrdU}^{+}$monocytes ${ }^{\dagger}$ & $8^{\dagger}$ & $96.2(72.8)$ & 6 & $33.5(23.5)^{* *}$ \\
\hline $\mathrm{CD}^{+} \mathrm{T}_{\text {cells }}$ & 11 & $152.2(418.4)$ & 6 & $200.1(89.8)$ \\
\hline
\end{tabular}

${ }^{*} P<0.05,{ }^{*} P<0.01$, and ${ }^{* * * P}<0.001$ for SIV/AIDS versus SIV + ART (nonparametric $U$-test).

${ }^{\dagger}$ No flow cytometry or BrdU data available for animals A20 to A22.

BrdU, bromodeoxyuridine; CCL, chemokine (C-C motif) ligand; DRG, dorsal root ganglia; IENFD, intraepidermal nerve fiber density; IQR, interquartile range; LDRG, lumbar DRG; MFI, mean fluorescent intensity.

with SIV/AIDS animals (peripheral blood monocytes and expression; $P=0.08$ ) (Table 3 ).

The percentage intermediate $\mathrm{CD} 14^{+} \mathrm{CD} 16^{+}$monocytes, nonclassic $\mathrm{CD}^{-} 4^{-} \mathrm{CD} 16^{+}$monocytes, and classic $\mathrm{CD} 14^{+} \mathrm{CD} 16^{-}$monocytes significantly differed in SIVinfected animals compared with SIV + ART animals (peripheral blood monocytes and expression; $P<0.01, P<0.05$, and $P<0.01$, respectively) (Table 3 ). Intermediate and nonclassic monocytes were significantly lower in SIV + ART animals compared with SIV/AIDS animals, whereas classic monocytes were greater in SIV + ART animals compared with SIV/AIDS animals. The mean fluorescent intensity of CD163 on intermediate $\mathrm{CD} 14^{+} \mathrm{CD} 16^{+}$monocytes was significantly lower for SIV + ART animals compared with SIV/AIDS animals (peripheral blood monocytes and expression; $P<0.01$ ) (Table 3).

IENFD and histologic analysis of immune cells were used to compare changes to peripheral neuron afferents and inflammation of the DRG in SIV infection. In terminal SIV/ AIDS animals, IENFD exhibited a negative percentage change from pre-infection to necropsy, showing loss of IENFD as a result of untreated SIV infection. There was a positive change in IENFD from pre-infection to necropsy in SIV + ART animals, which was significant when compared with terminal SIV/AIDS, showing an improvement in IENFD with intervention of ART (IENFD measurements; $P<0.05$ ) (Table 3). Of note, three animals did not show a positive change in IENFD or return to baseline IENFD, suggesting that ART may not fully ameliorate reduction in IENFD in SIV infection (Supplemental Figure S3). These data suggest similarities to the HIV population, which shows a subset of patients with persistent loss of IENFD and HIV-associated sensory neuropathy. The lumbar DRG of SIV/AIDS and SIV + ART animals were stained for $\mathrm{CD}^{+} 8^{+}$resident macrophages, $\mathrm{CD} 163^{+} \mathrm{M} 2$-like macrophages, Mac387 ${ }^{+}$M1-like monocytes, $\mathrm{BrdU}^{+}$newly divided monocytes, and $\mathrm{CD}^{+} \mathrm{T}$ cells, which were reported as cells per millimeter squared (lumbar DRG immune cell counts) (Table 3 ). $\mathrm{CD}^{+} 8^{+}$resident macrophages and $\mathrm{CD}^{+}$ $\mathrm{T}$ cells did not significantly differ between the two groups. $\mathrm{CD}_{163}{ }^{+}$macrophages were significantly lower in SIV + ART animals compared with SIV/AIDS animals (lumbar DRG immune cell counts; $P<0.05$ ) (Table 3). Mac387 ${ }^{+}$monocytes and $\mathrm{BrdU}^{+}$monocytes were also significantly lower in SIV + ART animals than SIV/AIDS animals (lumbar DRG immune cell counts; $P<0.001$ and $P<0.05$, respectively) (Table 3 ). Together, these data suggest ART reduces general inflammation seen in high viremia of SIV infection.

\section{Histologic Classification of DRG Neurons by} Immunoreactivity for NF200, TrkA, and IB4 in a Rhesus Macaque Model

Neuronal classes were identified as $\mathrm{NF}^{+} 20^{+}$neurons, IB4 ${ }^{+}$ nonpeptidergic neurons, and $\operatorname{TrkA}^{+}$peptidergic neurons 
(Supplemental Figure S4, A-C). More important, previous studies have shown overlap between the NF200 and IB4 population of neurons; however, for our comparisons, they will be referred to as separate populations, with the NF200 population representing myelinate neurons and the IB4 population representing nonpeptidergic neurons. ${ }^{45,46}$ The average neuronal diameter of the three populations was compared in uninfected animals, and values significantly differed from one another (analysis of variance $<0.01$ ) (Supplemental Figure S4D). The median diameter of $\mathrm{NF}^{2} 20^{+}$neurons was $51.28 \mu \mathrm{m}(\mathrm{IQR}=8.20 \mu \mathrm{m})$; $\mathrm{IB}^{+}$ neurons, $39.49 \mu \mathrm{m}(\mathrm{IQR}=3.70 \mu \mathrm{m})$; and TrkA $^{+}$neurons, $50.53 \mu \mathrm{m}(\mathrm{IQR}=4.24 \mu \mathrm{m})$. As expected and in agreement with previous reports, $\mathrm{TrkA}^{+}$and $\mathrm{IB}^{+}{ }^{+}$neurons tended to be smaller diameter neurons compared with $\mathrm{NF}^{2} 20^{+}$ neurons. ${ }^{8,11,12}$

\section{Nonpeptidergic and Peptidergic Neurons Atrophy with SIV Infection}

Cell body diameter was used to infer atrophy of $\mathrm{NF}^{2} 20^{+}$, $\mathrm{IB}^{+}$, and $\mathrm{TrkA}^{+}$neurons in SIV infection. All positively stained neurons in the DRG were measured for the largest point-to-point distance of the cell body. The average diameter of $\mathrm{NF}_{200}{ }^{+}$neurons was not significantly different between uninfected, terminal SIV/AIDS, and SIV-infected,
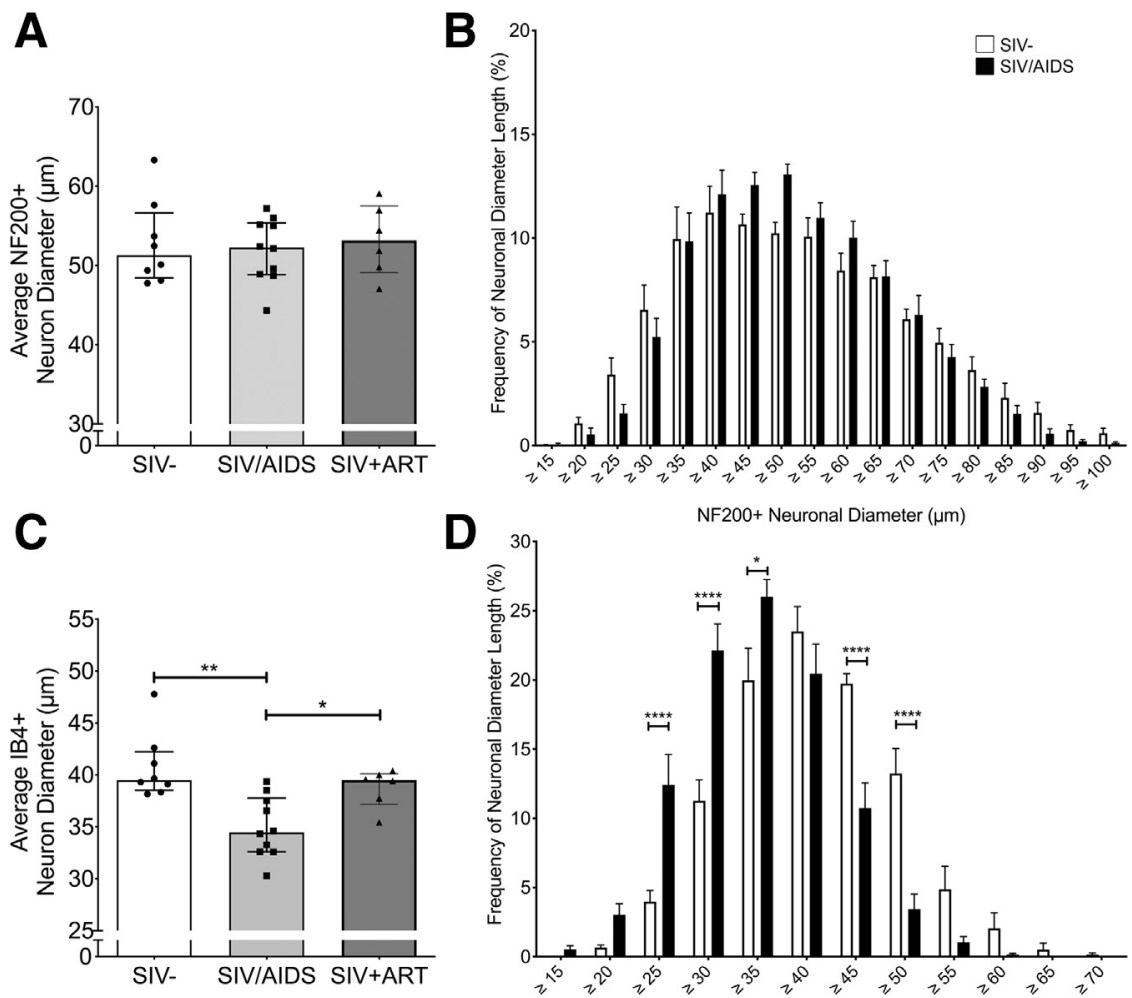

D
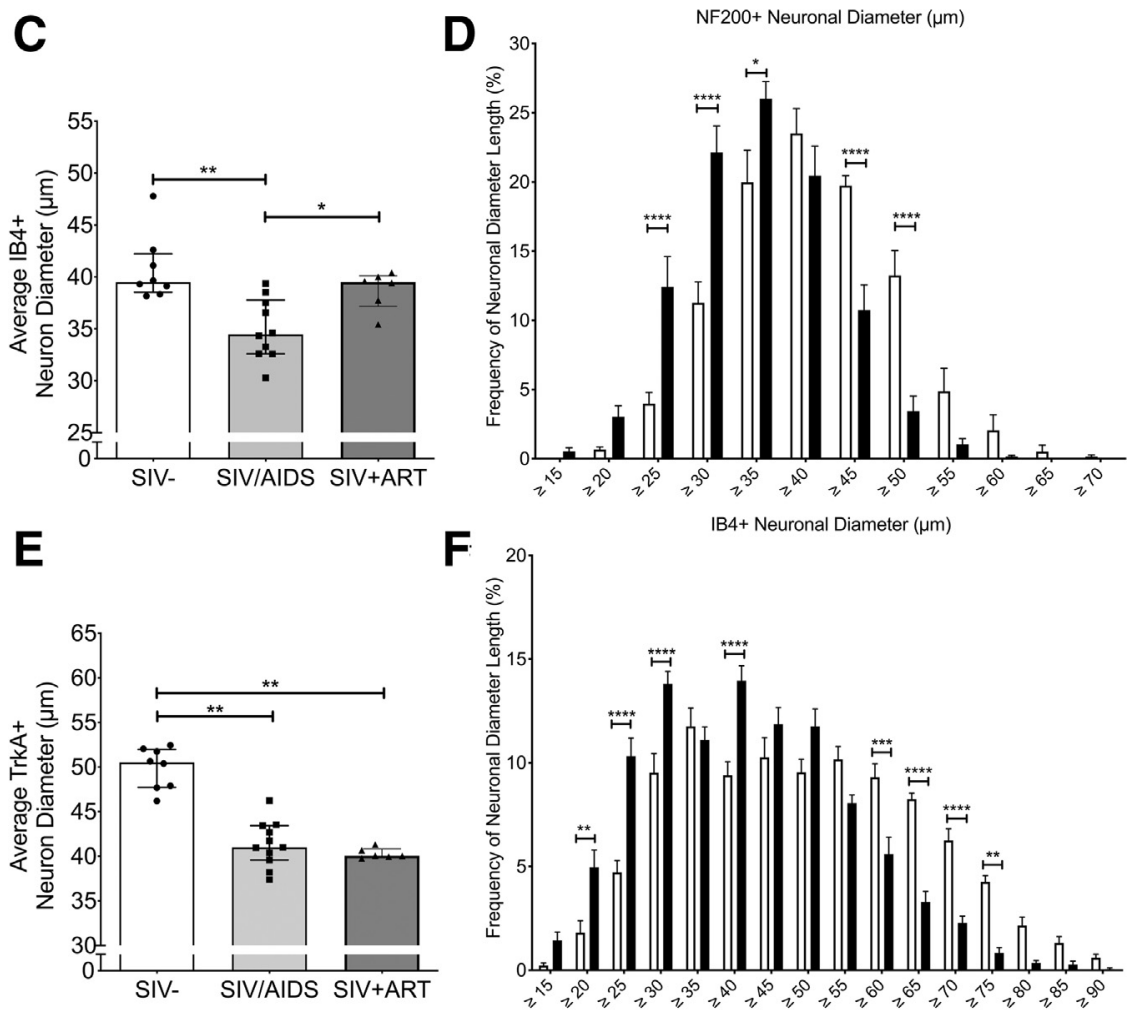

$\mathbf{F}$

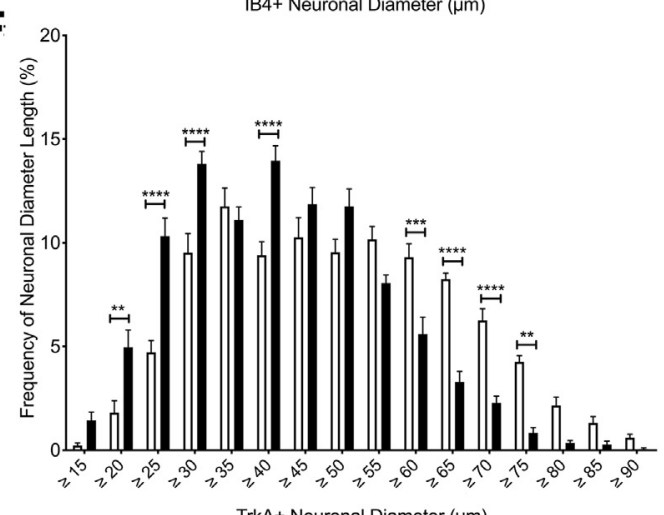

Figure 1 Nonpeptidergic and peptidergic nociceptive neurons atrophy in SIV infection. Neuronal diameters were measured as the longest point-to-point distance of the neuronal cell body of $\mathrm{NF}_{200}{ }^{+}, \mathrm{IB}^{+}$, and TrkA ${ }^{+}$neurons and compared between uninfected (SIV-; A01 to A08), terminal SIV/AIDS animals (SIV/AIDS; A12 to A22), and SIV-infected, antiretroviral therapy (ART)-treated animals (SIV + ART; A23 to A28) (Kruskal-Wallis test; Dunn multiple comparison post-hoc analysis). A: The average $\mathrm{NF}^{2} \mathrm{OO}^{+}$neuronal diameter did not differ between uninfected, terminal SIV/AIDS, or SIV + ART animals (analysis of variance). C: The average $\mathrm{IB}^{+}$neuronal diameter significantly differed between uninfected, terminal SIV/AIDS, and SIV + ART animals (analysis of variance; $P<0.01$ ). Terminal SIV/AIDS animals had smaller cell body diameter compared with uninfected and SIV + ART animals (Dunn post-hoc test; $P<0.01$ and $P<0.05$, respectively). E: The average $\mathrm{TrkA}^{+}$neuronal diameter significantly differed between uninfected, terminal SIV/AIDS, and SIV + ART animals (analysis of variance; $P<0.001)$, with terminal SIV/AIDS and SIV + ART animals having smaller diameters compared with uninfected animals (Dunn post-hoc test; $P<0.01$ and $P<0.01$, respectively). The frequency distributions were graphed and compared between uninfected and SIV-infected animals (two-way analysis of variance; Bonferroni multiple comparison post-hoc analysis). B: $\mathrm{NF}^{200^{+}}$neuronal diameter frequencies did not differ between uninfected and SIV/AIDS animals (two-way analysis of variance). D: $\mathrm{IB}^{+}{ }^{+}$neuronal diameter frequencies significantly differed between uninfected and SIV/ AIDS animals, with Bonferroni post-hoc significance of $5-\mu \mathrm{m}$ increments denoted on graph (twoway analysis of variance; $P<0.0001$ ). F: $\mathrm{TrkA}^{+}$neuronal diameter frequencies significantly differed between uninfected and SIV/AIDS animals, with Bonferroni post-hoc significance of 5$\mu \mathrm{m}$ increments denoted on graph (analysis of variance; $P<0.0001$ ). Post-hoc significance (Dunn or Bonferroni multiple comparison) was reported on graphs. Graphs represent the median value of each group with the interquartile range $(\mathbf{A}, \mathbf{C}$, and E). $n=9$ SIV $-(\mathbf{A}-\mathbf{F}) ; n=10$ SIV/AIDS $(\mathbf{A}-\mathbf{F})$; $n=6$ SIV + ART $(\mathbf{A}-\mathbf{F}) .{ }^{*} P<0.05,{ }^{*} P<0.01$ $* * * P<0.001$, and $* * * * P<0.0001$. 
ART-treated animals (Figure 1A). The average diameter of $\mathrm{IB}^{+}{ }^{+}$neurons significantly differed between uninfected, terminal SIV/AIDS, and SIV-infected, ART-treated animals (analysis of variance; $P<0.01$ ) (Figure $1 \mathrm{C}$ ), with significantly larger neuronal diameter for uninfected animals compared with terminal SIV/AIDS animals (post-hoc Dunn test; $P<0.01)$ and significantly larger neuronal diameter for SIV-infected, ART-treated animals compared with terminal SIV/AIDS (post-hoc Dunn test; $P<0.05$ ). TrkA ${ }^{+}$neuronal diameter also significantly differed between the three groups (analysis of variance; $P<0.001$ ) (Figure 1E), with significantly smaller diameters in terminal SIV/AIDS and SIV-infected, ART-treated animals compared with uninfected animals (post-hoc Dunn test; $P<0.01$ and $P<0.01$, respectively). The frequency of neuronal diameters was segregated by $5-\mu \mathrm{m}$ increments and used to identify global changes in neuronal diameter. Frequency distributions were compared between uninfected and terminal SIV/AIDS animals. The frequency distributions of neuronal diameters of $\mathrm{NF}^{2} \mathrm{OO}^{+}$neurons for uninfected and terminal SIV/AIDS did not significantly differ (two-way analysis of variance) (Figure 1B). The frequency distributions of $\mathrm{IB}^{+}{ }^{+}$neuron diameters (two-way analysis of variance; interaction $P<0.0001$; Bonferroni post-hoc analysis) (Figure 1D) and TrkA $^{+}$neuron diameters (two-way analysis of variance; interaction $P<0.0001$; Bonferroni post-hoc analysis) (Figure 1F) significantly differed between uninfected and terminal SIV/AIDS animals. The neuronal diameter was correlated to the viral load in terminal SIV/AIDS animals and SIV + ART animals, inferring changes associated with reduction of SIV viremia. The $\mathrm{NF}_{2} 00^{+}$and TrkA ${ }^{+}$neuronal diameter did not correlate with the plasma viral load, whereas the $\mathrm{IB}^{+}$neuronal diameter was negatively correlated to the plasma viral load (Supplemental Figure S5) [Spearman correlation; $P>0.05$ (Supplemental Figure S5A); $P=0.01, \mathrm{R}=-0.63$ (Supplemental Figure S5B); $P>0.05$ (Supplemental Figure S5C)]. These data show no significant difference in neuronal body size of $\mathrm{NF}^{2} \mathrm{OO}^{+}$neurons of uninfected and terminal SIV/ AIDS animals. Conversely, IB4 ${ }^{+}$and $\mathrm{TrkA}^{+}$neurons showed population-wide shift in the frequencies of neuronal diameters to a greater prevalence of smaller diameter in animals with SIV infection. In addition, reduction of viral load correlated to a larger diameter in the $\mathrm{IB} 4^{+}$neuronal population, suggesting an improved neuronal health with reduced viral load, but $\mathrm{TrkA}^{+}$neuronal diameter remained smaller even with reduction in viral load.

\section{Nonpeptidergic and Peptidergic Neurons Are Selectively Lost in SIV Infection}

The percentages of $\mathrm{NF}_{200}{ }^{+}$, IB $4^{+}$, and $\mathrm{TrkA}^{+}$neurons were compared among uninfected, SIV/DPI21, terminal SIV/AIDS, and SIV + ART animals. There was no observed difference in the percentage of $\mathrm{NF}^{2} 20^{+}$neurons between the four groups (Figure 2A). The percentage of $\mathrm{IB}^{+}{ }^{+}$neurons significantly differed among uninfected, SIV/ DPI21, terminal SIV/AIDS, and SIV + ART animals (analysis of variance $<0.01$ ) (Figure 2B), with a significantly lower percentage in SIV/AIDS animals compared with uninfected animals (post-hoc Dunn test; $P<0.01$ ). The percentage of TrkA ${ }^{+}$neurons significantly differed between uninfected, SIV/DPI21, terminal SIV/AIDS, and SIV + ART animals (analysis of variance $<0.01$ ) (Figure 2C). The percentage of TrkA ${ }^{+}$neurons was significantly less in SIV + ART animals compared with
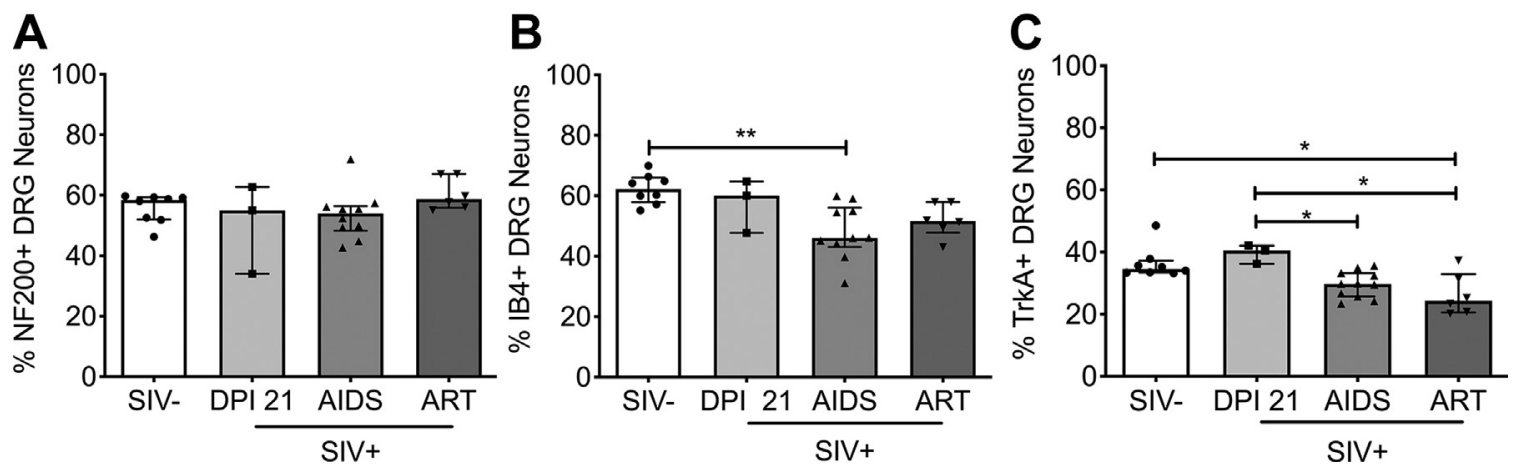

Figure 2 Loss of nonpeptidergic and peptidergic nociceptive neurons with SIV infection. Neuronal populations were quantified and reported as percentage positive neurons in uninfected (SIV-; A01 to A08), early SIV-infected (SIV/DPI21; A09 to A11), terminal SIV/AIDS (SIV/AIDS; A12 to A22), and SIV + antiretroviral therapy (ART) animals (SIV + ART; A23 to A28) (Kruskal-Wallis test; Dunn multiple comparison post-hoc test). A: The percentage of $\mathrm{NF}_{200^{+}}$neurons did not significantly differ between uninfected, early SIV-infected, terminal SIV/AIDS, and SIV + ART animals (analysis of variance). B: The percentage of $\mathrm{IB}^{+}$neurons significantly differed between uninfected, early SIV infection, terminal SIV/AIDS, and SIV + ART animals (analysis of variance; $P<0.01$ ), with a significantly lower percentage of IB4 ${ }^{+}$neurons in terminal SIV/AIDS compared with uninfected animals (Dunn post-hoc test; $\left.P<0.01\right)$. C: The percentage of TrkA ${ }^{+}$neurons significantly differed between uninfected, early SIV infection, terminal SIV/AIDS, and SIV + ART animals (analysis of variance; $P<0.01$ ). The percentage of TrkA + neurons was significantly less in SIV + ART compared with uninfected animals (Dunn post-hoc test; $P<0.05$ ), and significantly less in terminal SIV/AIDS and SIV + ART compared with SIV/days post infection (DPI) 21 animals (Dunn post-hoc test; $P<0.05$ and $P<0.05$, respectively). Post-hoc significance (Dunn multiple comparison) was reported. Graphs represent the median value of each group with the interquartile range $(\mathbf{A}-\mathbf{C}) . n=9 \mathrm{SIV}-(\mathbf{A}-\mathbf{C}) ; n=3 \mathrm{SIV} / \mathrm{DPI} 21(\mathbf{A}-\mathbf{C}) ; n=10 \mathrm{SIV} / \mathrm{AIDS}(\mathbf{A}-\mathbf{C}) ; n=6 \mathrm{SIV}+\mathrm{ART}(\mathbf{A}-\mathbf{C}) .{ }^{*} P<0.05, * * P<0.01$. 
uninfected animals (post-hoc Dunn test; $P<0.05$ ). Terminal SIV/AIDS and SIV + ART animals showed a significantly lower percentage of $\mathrm{TrkA}^{+}$neurons compared with SIV/DPI21 animals (post-hoc Dunn test; $P<0.05$ and $P<0.05$, respectively).

\section{Cleaved Caspase 3 Accumulates in DRG Neuronal Cell Bodies in SIV Infection}

Caspase 3 cleavage traditionally signifies a late apoptotic event and was used as a marker of end-stage sensory neuron apoptotic signaling. Relative caspase 3 mRNA was determined through RNAscope, and there was no observed difference in caspase $3 \mathrm{mRNA}$ expression in DRG neurons of uninfected, terminal SIV/AIDS, and SIV + ART animals (data not shown), suggesting that positive staining for cleaved caspase 3 protein was a result of increased proteolytic activation without increased caspase 3 mRNA expression. Cleaved caspase 3 protein was visualized as granular staining in the cytosol of neuronal cell bodies (Figure 3, A-D), and the percentage of cleaved caspase $3^{+}$ neurons was significantly different among uninfected, terminal SIV/AIDS, and SIV + ART animals (analysis of variance $<0.001 ;$ SIV/DPI21 not included in statistical analysis because $n=2$ ) (Figure 3E). Cleaved caspase $3^{+}$ neurons were significantly greater in SIV/AIDS animals compared with uninfected (post-hoc Dunn test; $P<0.01$ ) and SIV + ART animals (post-hoc Dunn test; $P<0.05$ ). There was no significant difference between uninfected and SIV + ART animals. The percentage of cleaved caspase $3^{+}$neurons was correlated to the average neuronal diameter (atrophy) in uninfected and terminal SIV/AIDS animals, to infer a correlation between atrophy and apoptotic events. There was no significant correlation between cleaved caspase $3^{+}$neurons and the average diameter of $\mathrm{NF}^{200}{ }^{+}$neurons (Spearman correlation; $P>0.05$ ) (Figure 3F). There was a significant negative correlation between the percentage of cleaved caspase $3^{+}$neurons and the average diameter of $\mathrm{IB}^{+}{ }^{+}$neurons (Spearman correlation; $P<0.01, R=-0.70$ ) (Figure 3G) and TrkA $^{+}$neurons (Spearman correlation; $P<0.001$, $R=-0.72$ ) (Figure $3 \mathrm{H}$ ). Of note, cleaved caspase 3 was also visualized in the axons of DRG neurons; however, DRG axons in tissue sections are indeterminate of innervation and could be innervating peripheral targets or the dorsal horn of the spinal column. Immunofluorescence for
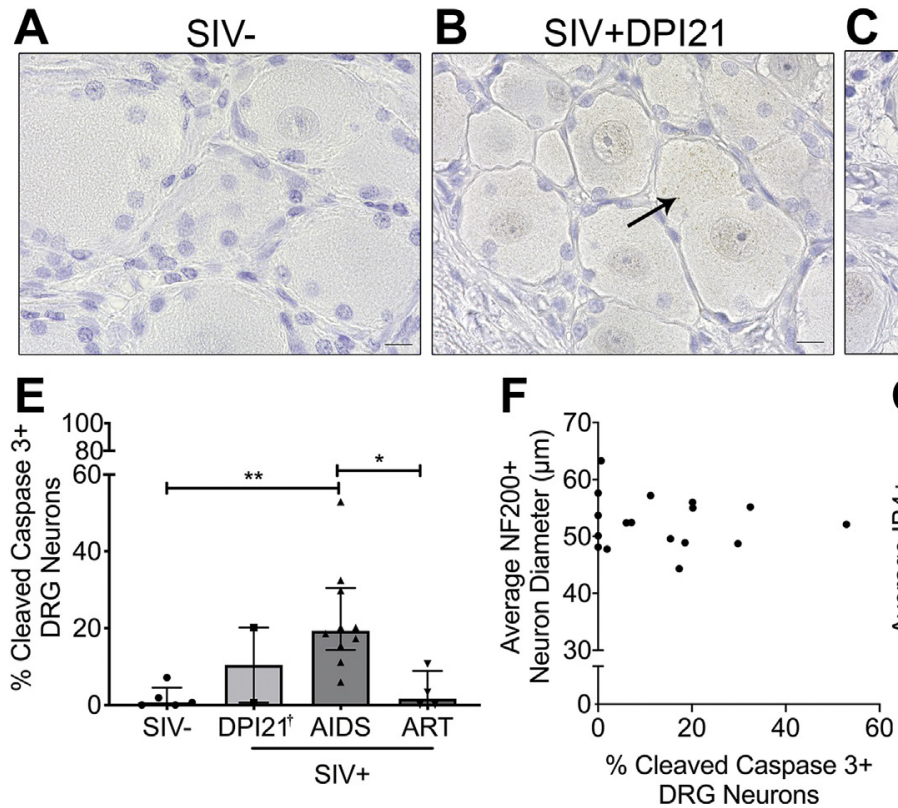
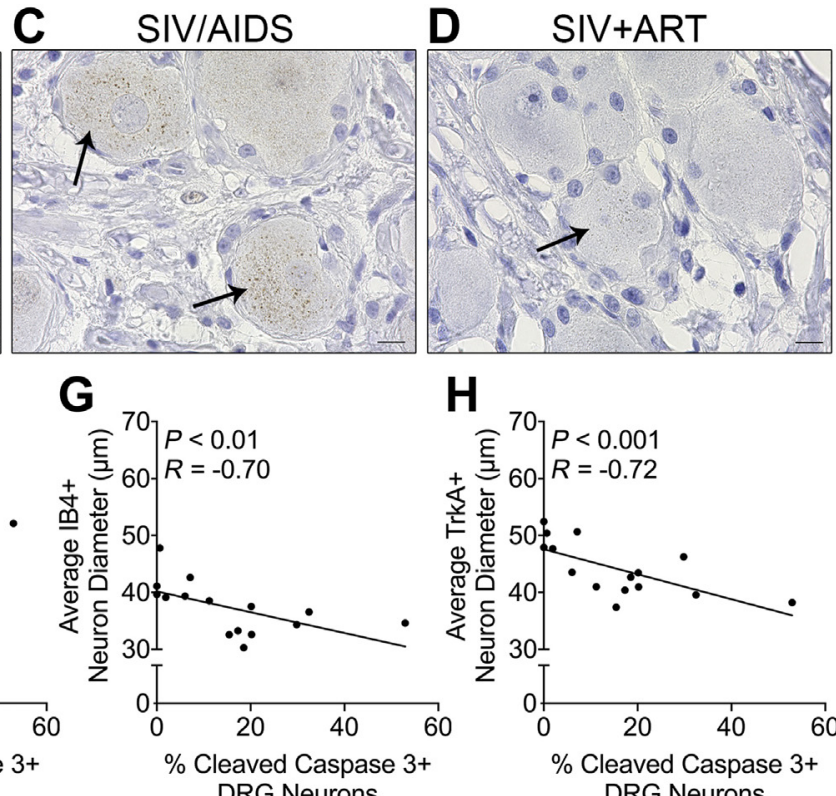

Figure 3 Accumulation of cytosolic cleaved caspase 3 in dorsal root ganglia (DRG) neurons with SIV infection. Cleaved caspase 3 protein (activated caspase 3) was visualized in DRG neuron cytosol and compared between uninfected (SIV_; A01 to A04 and A08), early SIV-infected (SIV/DPI21; A09 and A10), terminal SIV/AIDS (SIV/AIDS; A12 and A14 to A22), and SIV + antiretroviral therapy (ART) animals (SIV + ART; A23 to A24 and A26 to A28) (Kruskal-Wallis test; Dunn multiple comparison post-hoc test; SIV + DPI21 not included in analysis). A-D: Representative images show cytosolic granules of cleaved caspase 3 in DRG neurons (arrows). E: The percentage of cleaved caspase $3^{+}$neurons significantly differed between uninfected, terminals SIV/AIDS, and SIV + ART animals (analysis of variance; $P<0.001$ ), with significantly greater cleaved caspase $3^{+}$neurons in SIV/AIDS compared with uninfected and SIV + ART animals (Dunn post-hoc test; $P<0.01$ and $P<0.05$, respectively). The average neuronal cell body diameter of uninfected and terminal SIV/AIDS animals (denoting atrophy) was compared with the percentage of cleaved caspase $3^{+}$neurons (Spearman correlation). F: The diameter of NF200 ${ }^{+}$neurons did not significantly correlate to the percentage of cleaved caspase $3^{+}$neurons. $\mathbf{G}$ and $\mathbf{H}$ : The percentage of cleaved caspase $3^{+}$neurons was significantly, negatively correlated to the average $\mathrm{IB}_{4}^{+}$neuronal diameter $(P<0.01, \mathrm{R}=-0.70 ; \mathbf{G})$ and the average TrkA ${ }^{+}$neuronal diameter $(P<0.001, \mathrm{R}=-0.72 ; \mathbf{H})$. Post-hoc significance (Dunn multiple comparison) was reported on graphs. If Spearman correlation was significant, a linear regression (black lines) was added to graph for representation of correlational trend. †SIV/DPI21 was not included in statistical analysis because $n=2$. Graphs represent the median value of each group with the interquartile range $(\mathbf{E}) . n=5$ SIV $-(\mathbf{A}-\mathbf{H}) ; n=2$ SIV/DPI21 $(\mathbf{A}-\mathbf{H}) ; n=9$ SIV/AIDS $(\mathbf{A}-\mathbf{H}) ; n=5$ SIV + ART $(\mathbf{A}-\mathbf{H}) .{ }^{*} P<0.05, * * P<0.01$. Scale bars $=10 \mu \mathrm{m}(\mathbf{A}-\mathbf{D})$. Original magnification, $\times 100(\mathbf{A}-\mathbf{D})$. DPI, days post infection. 

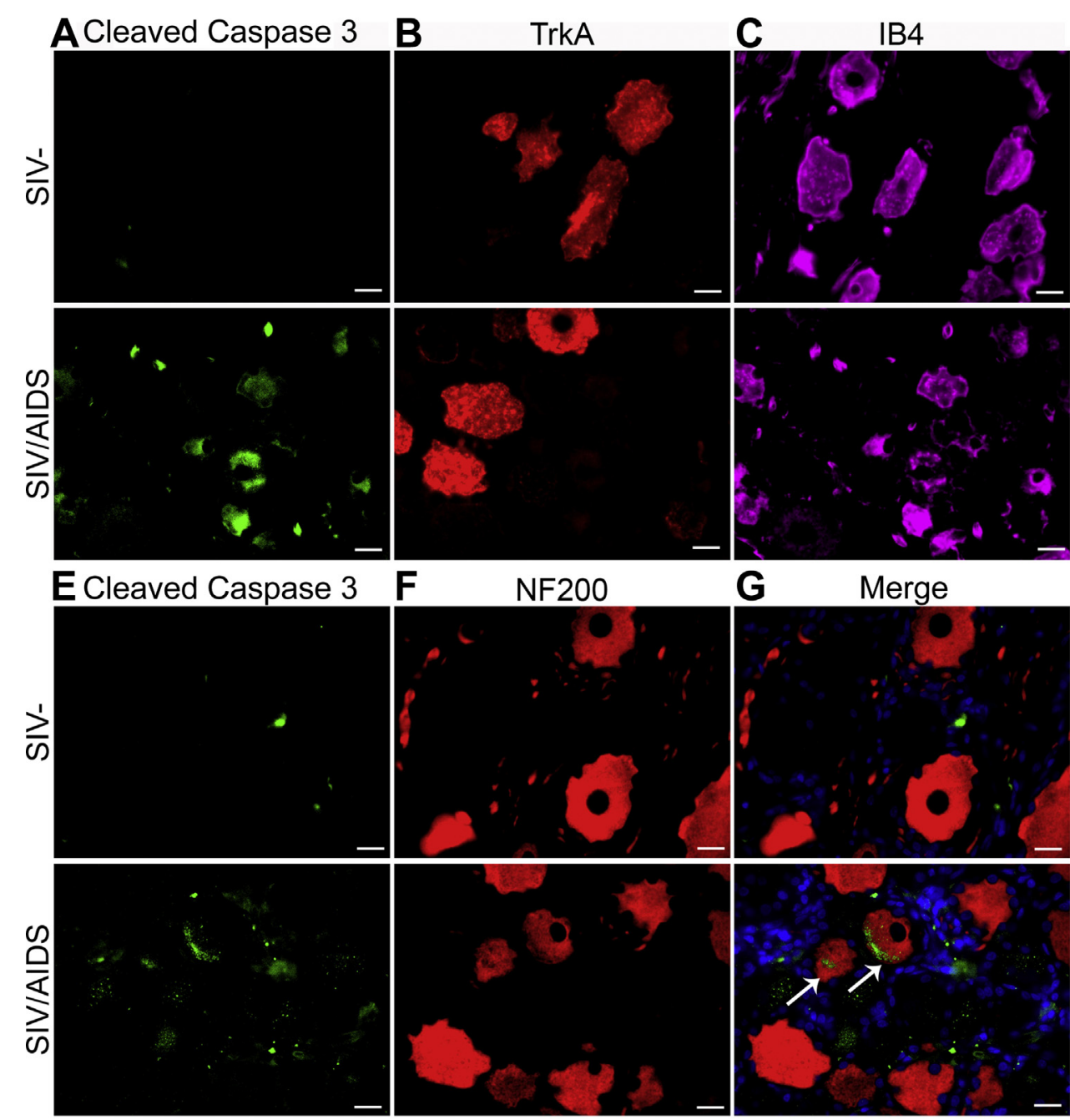

D Merge

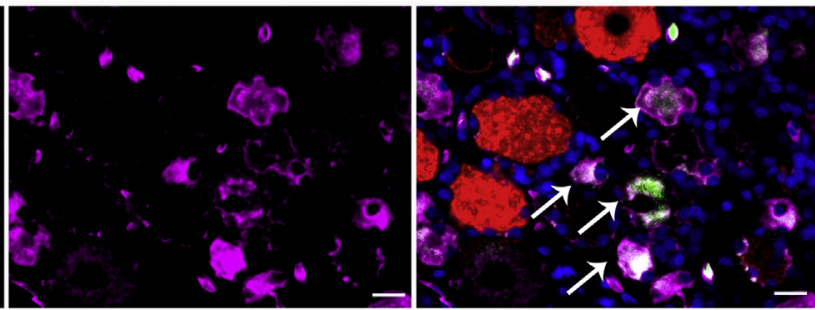

G Merge
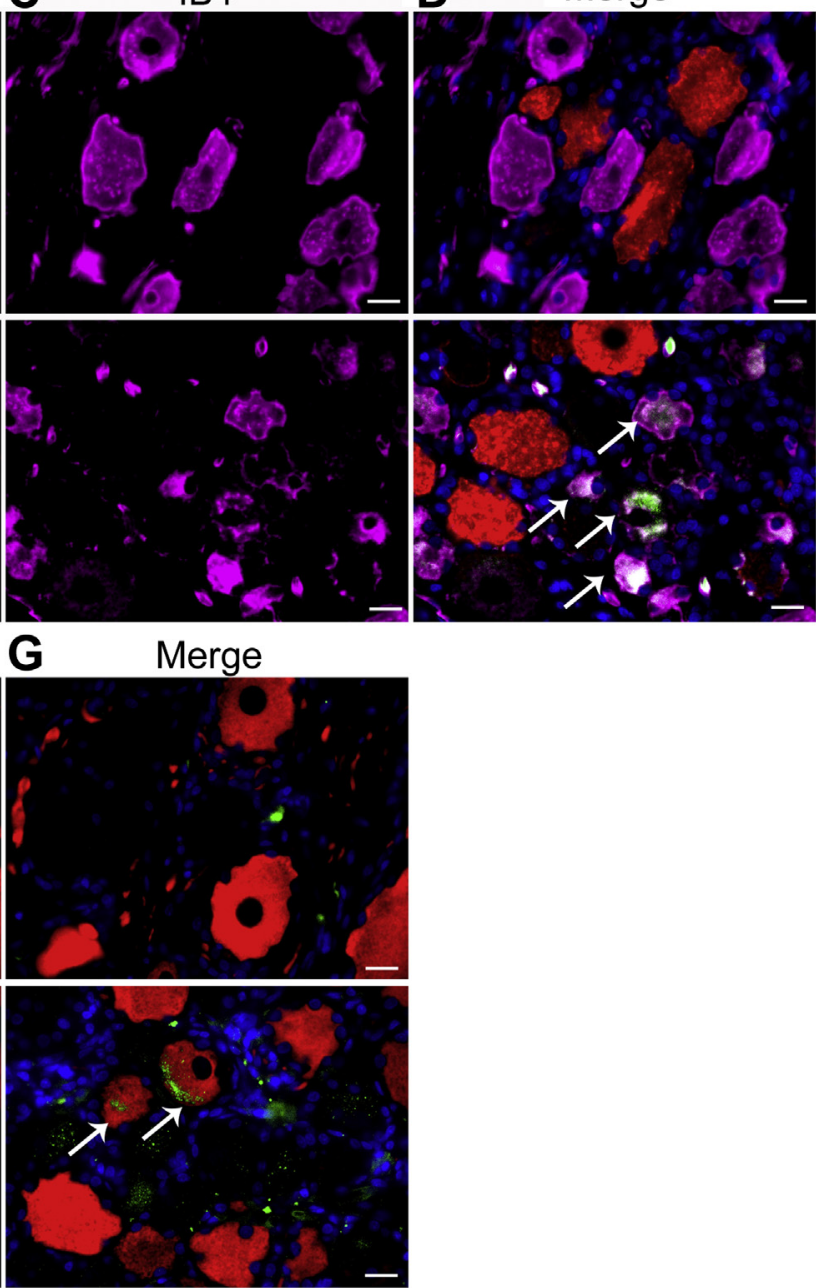

Figure 4 Cleaved caspase 3 colocalizes predominantly with nonpeptidergic sensory neurons. Immunofluorescence for cleaved caspase 3, NF200, IB4, and TrkA was used to identify the class of neurons with cytosolic cleaved caspase 3. Representative images of uninfected animals (SIV-) and terminal SIV/AIDS (SIV/AIDS) show cleaved caspase 3 (green; A), TrkA ${ }^{+}$neurons (red; B), IB4 ${ }^{+}$neurons (far red; C), and merged (D). In SIV/AIDS merged representative images, cleaved caspase $3^{+}$nonpeptidergic neurons are denoted with arrows. Representative images of SIV - and SIV/AIDS show cleaved caspase 3 (green; E), NF200 ${ }^{+}$ neurons (red; F), and merged $(\mathbf{G})$. In SIV/AIDS merged representative images, cleaved caspase $3^{+}$and NF200 neurons are denoted with arrows. Scale bars $=$ $20 \mu \mathrm{m}(\mathbf{A}-\mathbf{G})$. Original magnification, $\times 60(\mathbf{A}-\mathbf{G})$.

cleaved caspase 3, NF200, IB4, and TrkA was used to determine the colocalization of caspase 3 cascade activation in different classes of neurons in SIV-infected animals (Figure 4). Uninfected animals $(n=3)$ showed no positive staining for cleaved caspase 3 in the cell body of NF200 $\mathrm{IB}^{+}$, or TrkA ${ }^{+}$neurons (denoted SIV-) (Figure 4). Terminal SIV/AIDS animals showed a median of $9.74 \%$ $(\mathrm{IQR}=0.13 \% ; n=2)$ of $\mathrm{NF}^{200^{+}}$neurons colocalized with cleaved caspase $3,49.44 \%$ (IQR $=9.43 \% ; n=3$ ) of $\mathrm{IB}^{+}$neurons colocalized with cleaved caspase 3 , and $8.97 \%$ (IQR $=3.17 \% ; n=3$ ) of TrkA ${ }^{+}$neurons colocalized with cleaved caspase 3 (denoted SIV/AIDS) (Figure 4). Interestingly, IB4 ${ }^{+}$nonpeptidergic neurons showed a significant degree of overlap with cleaved cas-

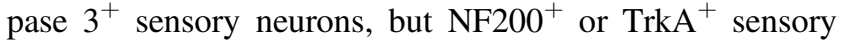
neurons showed less cleaved caspase 3 staining in the cell body. These data suggest that caspase 3 cascade activation is occurring largely in nonpeptidergic neurons with progression to terminal SIV/AIDS.

\section{ATF3 Accumulates in the Nucleus of Sensory Neurons during SIV Infection}

ATF3 is an integral response element for axon terminal regeneration in sensory neurons and was observed in the nucleus of DRG neurons (Figure 5, A-D). The percentage of neurons with $\mathrm{ATF}^{+}$nuclei was significantly different among uninfected, terminal SIV/AIDS, and SIV + ART animals (analysis of variance $<0.01$; SIV/DPI21 was not included in statistical analysis because $n=2$ ) (Figure 5E). The percentage of $\mathrm{ATF}^{+}$nuclei was significantly greater in terminal SIV/AIDS animals compared with uninfected animals $(P<0.01$; post-hoc test). There was no significant difference between SIV + ART animals and uninfected or 

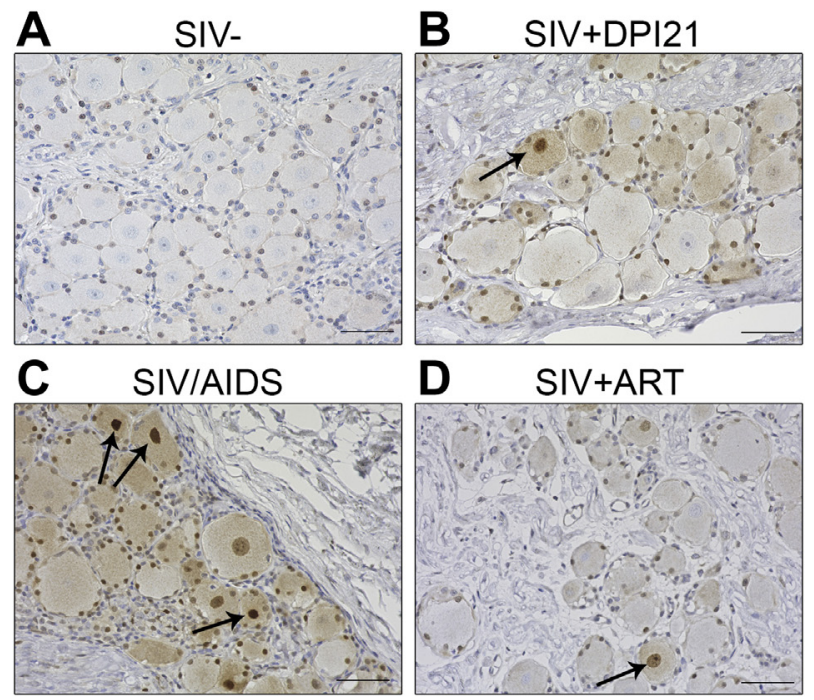

E

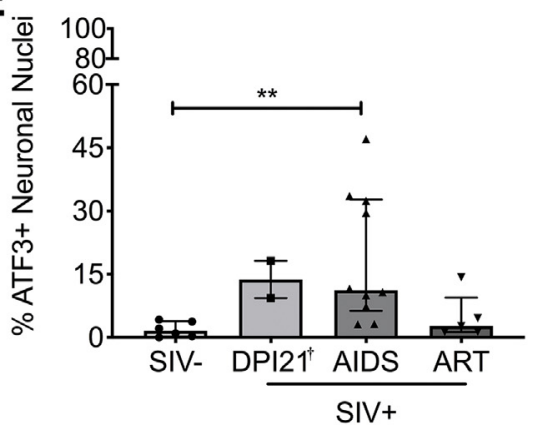

Figure 5 Increased nuclear activating transcription factor 3 (ATF3) in SIV infection. ATF3 was visualized in dorsal root ganglia neuron nucleus and compared between uninfected (SIV-; A01 to A04 and A06 to A08), early SIV-infected (SIV/DPI21; A09 and A10), terminal SIV/AIDS (SIV/ AIDS; A12 and A14 to A22), and SIV + ART animals (SIV + ART; A23, A24, and A26 to A28) (Kruskal-Wallis test; Dunn multiple comparison post-hoc test; SIV + DPI21 not included in analysis). A-D: Representative images show dense ATF3 immunoreactivity in the nucleus of positive neurons (arrows). E: The percentage of neurons with $\mathrm{ATF}^{+}{ }^{+}$nuclei was significantly different between uninfected, terminal SIV/AIDS, and SIV + ART animals (analysis of variance; $P<0.01$ ), with a significantly greater percentage of ATF3 $^{+}$neurons in SIV/AIDS animals compared with uninfected animals (Dunn post-hoc test; $P<0.01$ ). Post-hoc significance (Dunn multiple comparison) was reported on graphs. 'SIV/DPI21 was not included in statistical analysis because $n=2$. Graph represents the median value of each group with the interquartile range (E). $n=7$ SIV $-(\mathbf{A}-\mathbf{E}) ; n=2$ SIV/DPI21 (A-E); $n=9$ SIV/AIDS (A-E); $n=5$ SIV + ART (A-E). ${ }^{*} P<0.01$. Scale bar $=50 \mu \mathrm{m}(\mathbf{A}-\mathbf{D})$. Original magnification, $\times 40$ (A-D). ART, antiretroviral therapy; DPI, days post-infection.

terminal SIV/AIDS animals. Immunofluorescence for ATF3, NF200, IB4, and TrkA was used to determine the class of ATF3-expressing neurons in SIV-infected animals (Figure 6). Uninfected animals $(n=3)$ showed no positive staining for ATF3 in the neuronal nuclei of $\mathrm{NF}_{200^{+}}, \mathrm{IB} 4^{+}$, or $\mathrm{TrkA}^{+}$neurons (denoted SIV-) (Figure 6). Terminal SIV/AIDS animals showed a median of $3.43 \%$ $(\mathrm{IQR}=0.12 \% ; n=2)$ of $\mathrm{NF}_{200^{+}}$neuronal nuclei positive for ATF3, 7.07\% (IQR $=1.66 \% ; n=3$ ) of IB4 ${ }^{+}$ neuronal nuclei positive for ATF3, and $10.22 \%$ $(\mathrm{IQR}=8.99 \% ; n=3)$ of $\mathrm{TrkA}^{+}$neuronal nuclei positive for ATF3 (denoted SIV/AIDS) (Figure 6). Most ATF3 ${ }^{+}$ neurons were found to be either $\mathrm{IB}^{+}{ }^{+}$nonpeptidergic neurons or $\mathrm{TrkA}^{+}$peptidergic neurons in terminal SIV/ AIDS animals, with minimal overlap with $\mathrm{NF}_{200^{+}}$ neurons.

\section{Discussion}

Herein, we characterized damage to peripheral sensory neurons in an accelerated model of HIV infection with or without ART. Neuronal populations were classified as $\mathrm{NF}_{200^{+}}$myelinated neurons, IB4 ${ }^{+}$nonpeptidergic nociceptors, or TrkA ${ }^{+}$peptidergic nociceptors and assessed for neuronal atrophy, regeneration signaling, and activation of apoptosis in uninfected animals, early and terminal SIVinfected animals, and SIV-infected animals receiving a clinically relevant ART regimen. Successful reduction of viral load by ART dramatically reduced SIV-associated immune activation and DRG pathologic changes (viral load was not suppressed to undetectable). In the presence of ART, plasma inflammatory marker sCD14, sCD163, CCL2, and CCL5; monocyte expansion (percentage monocytes); inflammatory monocyte phenotypic shift (percentage $\mathrm{CD} 14^{+} \mathrm{CD} 16^{+}$, percentage $\mathrm{CD} 14^{-} \mathrm{CD} 16^{+}$, and $\mathrm{CD} 163$ mean fluorescent intensity); and myeloid infiltration of the DRG were all significantly lower, compared with untreated SIV infection. Over the course of infection, there was a significant improvement of IENFD, but three of the SIV + ART animals did not fully recover to baseline IENFD measurements. Taken together, ART may not fully remedy loss IENFD over the course of SIV infection.

As HIV-DSP presents with symmetric nociceptive pain, hypersensitivity to temperatures, and numbness or tingling in the extremities, we aimed to investigate disruption of nonpeptidergic and peptidergic neuron function and assessed the extent of sensory neuron damage in SIV infection. Using neuronal diameter as an indicator of atrophy and surrogate for neuronal dysfunction, a marked decline was observed in the average diameter of nonpeptidergic neurons in SIV infection; however, SIV-infected animals receiving ART had greater cell body diameters of nonpeptidergic neurons than terminal SIV/AIDS animals, showing a protective effect of viral suppression. Conversely, in the peptidergic neurons, significant atrophy was observed in both untreated and ART-treated, SIV-infected animal groups compared with uninfected control animals. Through comparison of total population diameter frequencies, nonpeptidergic and peptidergic neurons showed a populationwide shift in cell body size to that of a smaller diameter in terminal SIV/AIDS animals compared with uninfected control animals. In addition, comparison of the percentage of nonpeptidergic and peptidergic neurons between uninfected animals, SIV-infected animals, and SIV-infected animals receiving ART showed a selective loss of nociceptive neurons, with $\mathrm{NF}_{200}{ }^{+}$neurons being unaffected. More 


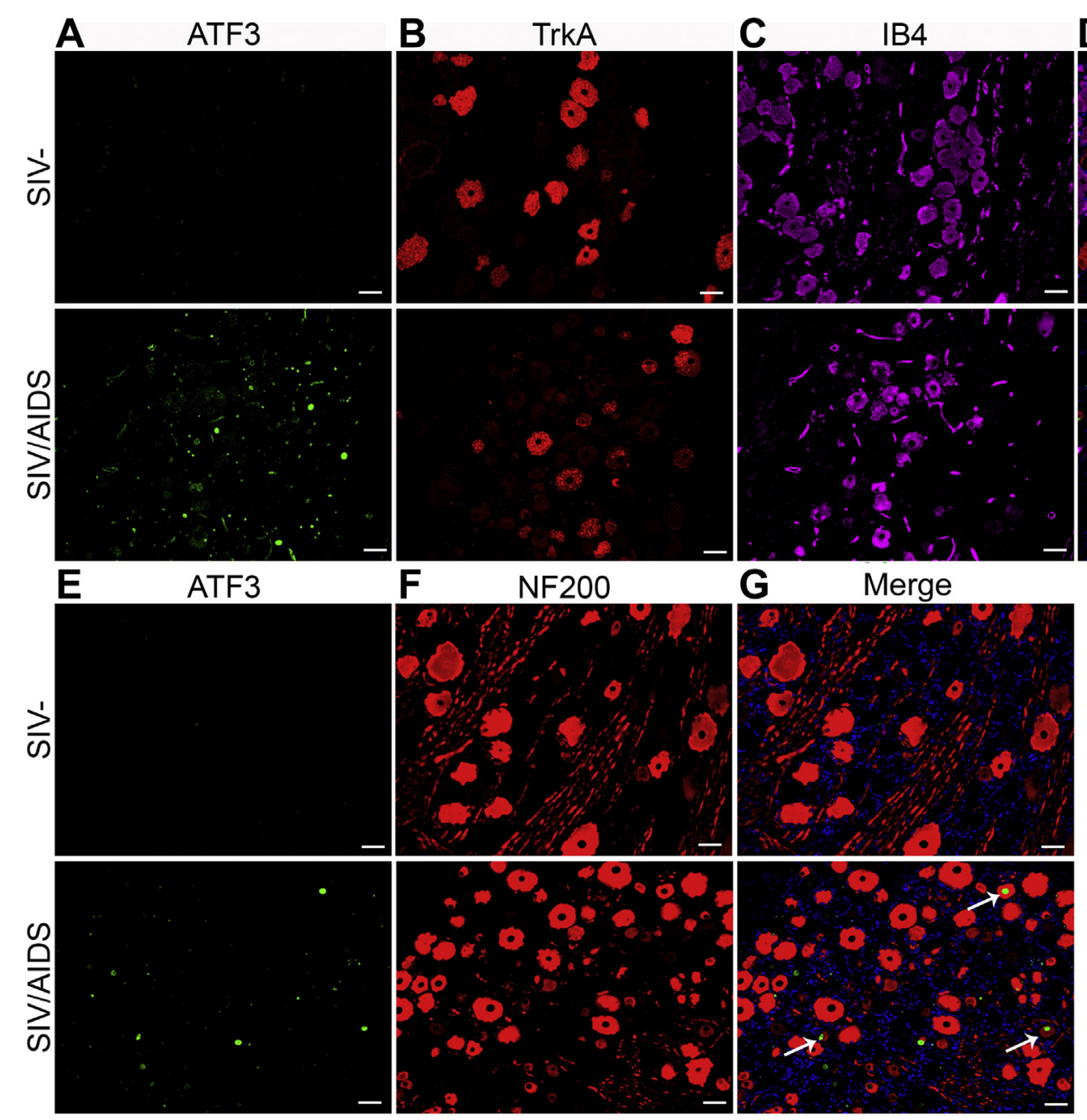

Figure 6 Activating transcription factor 3 (ATF3) localizes with nonpeptidergic and peptidergic neurons. Immunofluorescence for ATF3, NF200, IB4, and TrkA was used to identify the class of neurons with nuclear localization of ATF3. Representative images of uninfected animals (SIV-) and terminal SIV/AIDS (SIV/AIDS) show ATF3 ${ }^{+}$nuclei (green; A), TrkA ${ }^{+}$neurons (red; B), IB4 ${ }^{+}$neurons (far red; C), and merged (D). In SIV/AIDS merged representative images, $\mathrm{ATF}^{+}$nonpeptidergic and peptidergic neurons are denoted with arrows. Representative images of SIV - and SIV/AIDS show ATF $3^{+}$nuclei (green; E), NF200 ${ }^{+}$ neurons (red; F), and merged (G). In SIV/AIDS merged representative images, ATF3 ${ }^{+}$and NF200 ${ }^{+}$neurons are denoted with arrows. Scale bar $=50 \mu \mathrm{m}(\mathbf{A}-\mathbf{G})$. Original magnification, $\times 20(\mathbf{A}-\mathbf{G})$.

important, even with ART, peptidergic neurons remained significantly lower compared with uninfected and SIV/ DPI21 animals. Taken together, SIV infection is detrimental to nociceptive neurons and generates an unfavorable environment for both nonpeptidergic and peptidergic neurons. Intervention with ART may improve the pathologic changes observed in nonpeptidergic neurons; however, even with reduction in viremia and inflammation, peptidergic neurons seemed susceptible to atrophy, damage, and death.

Cleavage of caspase 3, an important effector caspase and traditional late-stage marker of apoptosis, was observed to different degrees in peripheral neurons in SIV infection. Caspase 3 is expressed in mature olfactory neurons throughout the cell bodies, axons, and synaptic terminals. ${ }^{47}$ On a damage event to sensory neurons, caspase 3 cleavage is initiated at the site of injury and, in the case of axotomy, procaspase 3 expression is significantly increased. ${ }^{47,48}$ No change was observed in caspase 3 expression in DRG neurons in SIV infection, suggesting increase in caspase 3 was the result of proteolytic processing. An increased number of cleaved caspase $3^{+}$neurons was observed in early SIV infection and was greater at terminal SIV/AIDS. Intervention with ART resulted in a lower number of cleaved caspase $3^{+}$neurons compared with untreated SIV infection. Interestingly, a significant portion of the $\mathrm{IB}^{+}{ }^{+}$ nonpeptidergic neurons $\left(49.44 \%\right.$ of $\mathrm{IB}^{+}{ }^{+}$neurons) colocalized with cleaved caspase 3 staining in SIV-infected animals, whereas $\mathrm{NF}^{2} 00^{+}$and $\mathrm{TrkA}^{+}$neurons colocalized to a lesser extent $(9.74 \%$ and $8.97 \%$, respectively). These data suggest that caspase 3-mediated mechanisms of apoptosis may be more pronounced in the IB4 nonpeptidergic neurons. In this study, a characteristic marker of late-stage apoptosis was used, but, other mechanisms of cell death, such as autophagy or necrosis, were not investigated, 
which may be more pertinent to TrkA ${ }^{+}$peptidergic neurons. Although caspase 3 was used to infer apoptotic signaling, peripheral neurons in a model of long-term experimental diabetic neuropathy showed accumulation of cleaved caspase 3 in the cell body, but the increased caspase 3 activation was not associated with cell death, ${ }^{49}$ suggesting a possible nonapoptotic mechanism of caspase 3 in sensory neurons. Cleaved caspase 3 was quantified in the neuronal cell body, but the initial caspase activation may not have occurred in the cell body, as cleaved caspase 3 was also observed in the axons of peripheral neurons. Further studies are needed to address the source of caspase 3 activation in peripheral neurons during SIV infection, whether it be at the cell body or axon, to identify possible nonapoptotic roles of caspase 3, to determine a threshold for apoptosis in sensory neurons, and to address contributions of other cell death mechanisms.

Peripheral sensory neurons have both a dynamic and direct interaction with body, making them particularly susceptible to changes in disease states, and in HIV-DSP, patients present with significant and progressive loss of IENFD. We investigated expression changes in ATF3, an integral transcription factor of the regeneration program in sensory neurons. In SIV infection, there was a greater number of DRG neurons with ATF3 localized to the nucleus, suggesting an induction of regeneration-associated gene transcripts. Although this is a cross-sectional study, ATF3 accumulation may be transient over the course of infection. In addition, neurons with high degree of nuclear ATF3 were identified to be mostly $\mathrm{IB}^{+}{ }^{+}$nonpeptidergic or $\mathrm{TrkA}^{+}$peptidergic neurons $(7.07 \%$ and $10.22 \%$, respectively), with $\mathrm{NF} 200^{+}$neurons colocalizing to a lesser extent $(3.43 \%)$. These findings support the notion that loss of IENFs of sensory neurons may promote regenerative signaling in nonpeptidergic and peptidergic neurons. Although we looked at a specific transcription factor, further studies are needed to identify synergism with other transcription factors and the involvement of specific proregenerative proteins and transcripts in the presence of SIV infection.

In summary, SIV infection generates an environment that drives neuropathologic changes in the DRG, with a classspecific impact on nonpeptidergic and peptidergic neurons. Loss of nonpeptidergic neurons in SIV infection was lessened by intervention with ART; however, peptidergic neurons appeared particularly susceptible to cell death and atrophy, in both the absence and the presence of ART. Future investigations should determine and compare resilience of nonpeptidergic and peptidergic neurons in different pathophysiological conditions, such as axotomy, inflammation, and infection. Although reduction was observed in cleaved caspase 3 and ATF3 with viral load reduction, subtler changes in nonpeptidergic and peptidergic neurons, such as expression of channel proteins, neuropeptides, and cytokines, were not studied. Further studies are needed to address long-term changes in nonpeptidergic and peptidergic neurons in the context of successful viral suppression and chronic, low-grade inflammation, giving insight into mechanisms of hypersensitivity, damage to peripheral axon terminals, and class-specific vulnerability to changes during HIV infection.

\section{Acknowledgments}

We thank Merck and Gilead for the antiretroviral therapy drugs used in this study; veterinary staff at the New England and Tulane National Primate Research Center for animal care; pathology residents and staff for assisting with necropsies and tissue collection; and Dr. Xavier Alvarez and research technician Cecily Midkiff for assistance on this project.

\section{Author Contributions}

This project was designed and conceived by T.H.B; data were acquired and analyzed by J.A.R., G.G., R.W., M.D.S., and J.R.L; pathology was assessed by A.D.M.; statistical analysis and interpretation were performed by J.A.R., G.G., R.W., J.R.L., M.S., and T.H.B; the manuscript was drafted by J.A.R. and T.H.B and critical revisions performed by J.A.R., A.D.M., and T.H.B.

\section{Supplemental Data}

Supplemental material for this article can be found at http://doi.org/10.1016/j.ajpath.2020.03.004.

\section{References}

1. Aziz-Donnelly A, Harrison TB: Update of HIV-associated sensory neuropathies. Curr Treat Options Neurol 2017, 19:36

2. Ellis RJ, Rosario D, Clifford DB, McArthur JC, Simpson D, Alexander T, Gelman BB, Vaida F, Collier A, Marra CM, Ances B, Atkinson JH, Dworkin RH, Morgello S, Grant I; CHARTER Study Group: Continued high prevalence and adverse clinical impact of human immunodeficiency virus-associated sensory neuropathy in the era of combination antiretroviral therapy: the CHARTER Study. Arch Neurol 2010, 67:552-558

3. Nicholas PK, Mauceri L, Slate Ciampa A, Corless IB, Raymond N, Barry DJ, Viamonte Ros A: Distal sensory polyneuropathy in the context of HIV/AIDS. J Assoc Nurses AIDS Care 2007, 18:32-40

4. Wulff EA, Wang AK, Simpson DM: HIV-associated peripheral neuropathy: epidemiology, pathophysiology and treatment. Drugs 2000, 59:1251-1260

5. McArthur JC, Brew BJ, Nath A: Neurological complications of HIV infection. Lancet Neurol 2005, 4:543-555

6. Pardo CA, McArthur JC, Griffin JW: HIV neuropathy: insights in the pathology of HIV peripheral nerve disease. J Peripher Nerv Syst 2001, 6:21-27

7. Polydefkis M, Yiannoutsos CT, Cohen BA, Hollander H, Schifitto G, Clifford DB, Simpson DM, Katzenstein D, Shriver S, Hauer P, Brown A, Haidich AB, Moo L, McArthur JC: Reduced intraepidermal nerve fiber density in HIV-associated sensory neuropathy. Neurology 2002, 58:115-119 
8. Liu M, Willmott NJ, Michael GJ, Priestley JV: Differential pH and capsaicin responses of Griffonia simplicifolia IB4 (IB4)-positive and IB4-negative small sensory neurons. Neuroscience 2004, 127: 659-672

9. McCoy ES, Taylor-Blake B, Street SE, Pribisko AL, Zheng J, Zylka MJ: Peptidergic CGRP $\alpha$ primary sensory neurons encode heat and itch and tonically suppress sensitivity to cold. Neuron 2013, 78 : $138-151$

10. Le Pichon CE, Chesler AT: The functional and anatomical dissection of somatosensory subpopulations using mouse genetics. Front Neuroanat 2014, 8:21

11. Averill S, McMahon SB, Clary DO, Reichardt LF, Priestley JV: Immunocytochemical localization of trkA receptors in chemically identified subgroups of adult rat sensory neurons. Eur J Neurosci 1995, 7:1484-1494

12. Molliver DC, Radeke MJ, Feinstein SC, Snider WD: Presence or absence of TrkA protein distinguishes subsets of small sensory neurons with unique cytochemical characteristics and dorsal horn projections. J Comp Neurol 1995, 361:404-416

13. Stucky CL, Lewin GR: Isolectin B(4)-positive and -negative nociceptors are functionally distinct. J Neurosci 1999, 19:6497-6505

14. Ebenezer GJ, Hauer P, Gibbons C, McArthur JC, Polydefkis M: Assessment of epidermal nerve fibers: a new diagnostic and predictive tool for peripheral neuropathies. J Neuropathol Exp Neurol 2007, 66:1059-1073

15. Ebenezer GJ, McArthur JC, Thomas D, Murinson B, Hauer P, Polydefkis M, Griffin JW: Denervation of skin in neuropathies: the sequence of axonal and Schwann cell changes in skin biopsies. Brain 2007, 130:2703-2714

16. Zylka MJ, Rice FL, Anderson DJ: Topographically distinct epidermal nociceptive circuits revealed by axonal tracers targeted to Mrgprd. Neuron 2005, 45:17-25

17. Lallemend F, Ernfors P: Molecular interactions underlying the specification of sensory neurons. Trends Neurosci 2012, 35: 373-381

18. Laast VA, Shim B, Johanek LM, Dorsey JL, Hauer PE, Tarwater PM, Adams RJ, Pardo CA, McArthur JC, Ringkamp M, Mankowski JL: Macrophage-mediated dorsal root ganglion damage precedes altered nerve conduction in SIV-infected macaques. Am J Pathol 2011, 179: $2337-2345$

19. Mangus LM, Weinberg RL, Knight AC, Queen SE, Adams RJ, Mankowski JL: SIV-induced immune activation and metabolic alterations in the dorsal root ganglia during acute infection. J Neuropathol Exp Neurol 2019, 78:78-87

20. Ebenezer GJ, Laast VA, Dearman B, Hauer P, Tarwater PM, Adams RJ, Zink MC, McArthur JC, Mankowski JL: Altered cutaneous nerve regeneration in a simian immunodeficiency virus/macaque intracutaneous axotomy model. J Comp Neurol 2009, 514: $272-283$

21. Lakritz JR, Robinson JA, Polydefkis MJ, Miller AD, Burdo TH: Loss of intraepidermal nerve fiber density during SIV peripheral neuropathy is mediated by monocyte activation and elevated monocyte chemotactic proteins. J Neuroinflammation 2015, 12:237

22. Makwana M, Raivich G: Molecular mechanisms in successful peripheral regeneration. FEBS J 2005, 272:2628-2638

23. van Kesteren RE, Mason MR, Macgillavry HD, Smit AB, Verhaagen J: A gene network perspective on axonal regeneration. Front Mol Neurosci 2011, 4:46

24. Tsujino H, Kondo E, Fukuoka T, Dai Y, Tokunaga A, Miki K, Yonenobu K, Ochi T, Noguchi K: Activating transcription factor 3 (ATF3) induction by axotomy in sensory and motoneurons: a novel neuronal marker of nerve injury. Mol Cell Neurosci 2000, 15: $170-182$

25. Seijffers R, Mills CD, Woolf CJ: ATF3 increases the intrinsic growth state of DRG neurons to enhance peripheral nerve regeneration. J Neurosci 2007, 27:7911-7920
26. Lindå H, Sköld MK, Ochsmann T: Activating transcription factor 3, a useful marker for regenerative response after nerve root injury. Front Neurol 2011, 2:30

27. Gey M, Wanner R, Schilling C, Pedro MT, Sinske D, Knöll B: Atf3 mutant mice show reduced axon regeneration and impaired regeneration-associated gene induction after peripheral nerve injury. Open Biol 2016, 6:160091

28. Fagoe ND, Attwell CL, Kouwenhoven D, Verhaagen J, Mason MR: Overexpression of ATF3 or the combination of ATF3, c-Jun, STAT3 and Smad1 promotes regeneration of the central axon branch of sensory neurons but without synergistic effects. Hum Mol Genet 2015, 24:6788-6800

29. Chandran V, Coppola G, Nawabi H, Omura T, Versano R, Huebner EA, Zhang A, Costigan M, Yekkirala A, Barrett L, Blesch A, Michaelevski I, Davis-Turak J, Gao F, Langfelder P, Horvath S, He Z, Benowitz L, Fainzilber M, Tuszynski M, Woolf CJ, Geschwind DH: A systems-level analysis of the peripheral nerve intrinsic axonal growth program. Neuron 2016, 89:956-970

30. Lakritz JR, Bodair A, Shah N, O'Donnell R, Polydefkis MJ, Miller AD, Burdo TH: Monocyte traffic, dorsal root ganglion histopathology, and loss of intraepidermal nerve fiber density in SIV peripheral neuropathy. Am J Pathol 2015, 185:1912-1923

31. Lakritz JR, Thibault DM, Robinson JA, Campbell JH, Miller AD, Williams KC, Burdo TH: $a 4$-Integrin antibody treatment blocks monocyte/macrophage traffic to, vascular cell adhesion molecule-1 expression in, and pathology of the dorsal root ganglia in an SIV macaque model of HIV-peripheral neuropathy. Am J Pathol 2016, 186:1754-1761

32. Dorsey JL, Mangus LM, Hauer P, Ebenezer GJ, Queen SE, Laast VA, Adams RJ, Mankowski JL: Persistent peripheral nervous system damage in simian immunodeficiency virus-infected macaques receiving antiretroviral therapy. J Neuropathol Exp Neurol 2015, 74: $1053-1060$

33. Burdo TH, Orzechowski K, Knight HL, Miller AD, Williams K: Dorsal root ganglia damage in SIV-infected rhesus macaques: an animal model of HIV-induced sensory neuropathy. Am J Pathol 2012, 180:1362-1369

34. Mangus LM, Dorsey JL, Laast VA, Ringkamp M, Ebenezer GJ, Hauer P, Mankowski JL: Unraveling the pathogenesis of HIV peripheral neuropathy: insights from a simian immunodeficiency virus macaque model. ILAR J 2014, 54:296-303

35. Burdo TH, Miller AD: Animal models of HIV peripheral neuropathy. Future Virol 2014, 9:465-474

36. Mangus LM, Rao DB, Ebenezer GJ: Intraepidermal nerve fiber analysis in human patients and animal models of peripheral neuropathy: a comparative review. Toxicol Pathol 2020, 48:59-70

37. Burdo TH, Soulas C, Orzechowski K, Button J, Krishnan A, Sugimoto C, Alvarez X, Kuroda MJ, Williams KC: Increased monocyte turnover from bone marrow correlates with severity of SIV encephalitis and CD163 levels in plasma. PLoS Pathog 2010, 6:e1000842

38. Whitney JB, Hill AL, Sanisetty S, Penaloza-MacMaster P, Liu J, Shetty M, Parenteau L, Cabral C, Shields J, Blackmore S, Smith JY, Brinkman AL, Peter LE, Mathew SI, Smith KM, Borducchi EN, Rosenbloom DI, Lewis MG, Hattersley J, Li B, Hesselgesser J, Geleziunas R, Robb ML, Kim JH, Michael NL, Barouch DH: Rapid seeding of the viral reservoir prior to SIV viraemia in rhesus monkeys. Nature 2014, 512:74-77

39. Del Prete GQ, Smedley J, Macallister R, Jones GS, Li B, Hattersley J, Zheng J, Piatak M, Keele BF, Hesselgesser J, Geleziunas R, Lifson JD: Short communication: comparative evaluation of coformulated injectable combination antiretroviral therapy regimens in simian immunodeficiency virus-infected rhesus macaques. AIDS Res Hum Retroviruses 2016, 32:163-168

40. Kearns AC, Robinson JA, Shekarabi M, Liu F, Qin X, Burdo TH: Caspase-1-associated immune activation in an accelerated SIVinfected rhesus macaque model. J Neurovirol 2018, 24:420-431 
41. Mangus LM, Dorsey JL, Laast VA, Hauer P, Queen SE, Adams RJ, McArthur JC, Mankowski JL: Neuroinflammation and virus replication in the spinal cord of simian immunodeficiency virus-infected macaques. J Neuropathol Exp Neurol 2015, 74:38-47

42. Campbell JH, Ratai EM, Autissier P, Nolan DJ, Tse S, Miller AD, González RG, Salemi M, Burdo TH, Williams KC: Anti- $\alpha 4$ antibody treatment blocks virus traffic to the brain and gut early, and stabilizes CNS injury late in infection. PLoS Pathog 2014, 10:e1004533

43. Zanni MV, Toribio M, Wilks MQ, Lu MT, Burdo TH, Walker J, Autissier P, Foldyna B, Stone L, Martin A, Cope F, Abbruzzese B, Brady T, Hoffmann U, Williams KC, El-Fakhri G, Grinspoon SK: Application of a novel CD206+ macrophage-specific arterial imaging strategy in HIV-infected individuals. J Infect Dis 2017, 215:1264-1269

44. Lakritz JR, Yalamanchili S, Polydefkis MJ, Miller AD, McGrath MS, Williams KC, Burdo TH: An oral form of methylglyoxal-bisguanylhydrazone reduces monocyte activation and traffic to the dorsal root ganglia in a primate model of HIV-peripheral neuropathy. J Neurovirol 2017, 23:568-576
45. Vukojevic K, Filipovic N, Tica Sedlar I, Restovic I, Bocina I, Pintaric I, Saraga-Babic M: Neuronal differentiation in the developing human spinal ganglia. Anat Rec (Hoboken) 2016, 299: 1060-1072

46. Russo D, Clavenzani P, Mazzoni M, Chiocchetti R, Di Guardo G, Lalatta-Costerbosa G: Immunohistochemical characterization of TH13-L2 spinal ganglia neurons in sheep (Ovis aries). Microsc Res Tech 2010, 73:128-139

47. Cowan CM, Thai J, Krajewski S, Reed JC, Nicholson DW, Kaufmann SH, Roskams AJ: Caspases 3 and 9 send a pro-apoptotic signal from synapse to cell body in olfactory receptor neurons. J Neurosci 2001, 21:7099-7109

48. Hu G, Huang K, Hu Y, Du G, Xue Z, Zhu X, Fan G: Single-cell RNA-seq reveals distinct injury responses in different types of DRG sensory neurons. Sci Rep 2016, 6:31851

49. Cheng C, Zochodne DW: Sensory neurons with activated caspase-3 survive long-term experimental diabetes. Diabetes 2003, 52: $2363-2371$ 